Article

\title{
Evaluating Soybean Cultivars for Low- and High-Temperature Tolerance During the Seedling Growth Stage
}

\author{
Firas Ahmed Alsajri ${ }^{1}$, Bhupinder Singh ${ }^{2}$, Chathurika Wijewardana ${ }^{1}$, J. Trenton Irby ${ }^{1}$, \\ Wei Gao ${ }^{3}$ and Kambham Raja Reddy ${ }^{1, * \mathbb{D}}$ \\ 1 Department of Plant and Soil Sciences, Mississippi State University, Starkville, MS 39762, USA; \\ faa34@msstate.edu (F.A.A.); gkw37@msstate.edu (C.W.); tirby@pss.msstate.edu (J.T.I.) \\ 2 Delta Research and Extension Center, Mississippi State University, Stoneville, MS 38776, USA; \\ bs1725@msstate.edu \\ 3 USDA-UVB Monitoring and Research Program, Natural Resource Ecology Laboratory, and Department of \\ Ecosystem Science and Sustainability, Colorado State University, Fort Collins, CO 80523, USA; \\ Wei.Gao@colostate.edu \\ * Correspondence: krreddy@pss.msstate.edu; Fax.: +1-662-325-9461
}

Received: 1 December 2018; Accepted: 22 December 2018; Published: 1 January 2019

\begin{abstract}
Soybean (Glycine max L.) seedlings may be exposed to low or high temperatures under early or conventional soybean production systems practiced in the US Midsouth. However, a wide range of soybean cultivars commonly grown in the region may inherit diverse tolerance to degrees of temperatures. Therefore, a study was conducted in a controlled-environment facility to quantify 64 soybean cultivars from Maturity Group III to V, to low (LT; 20/12 $\left.{ }^{\circ} \mathrm{C}\right)$, optimum (OT; $\left.30 / 22{ }^{\circ} \mathrm{C}\right)$, and high $\left(\mathrm{HT} ; 40 / 32^{\circ} \mathrm{C}\right)$ temperature treatments during the seedling growth stage. Several shoot, root, and physiological parameters were assessed at 20 days after sowing. The study found a significant decline in the measured root, shoot, and physiological parameters at both low and high temperatures, except for root average diameter (RAD) and lateral root numbers under LT effects. Under HT, shoot growth was significantly increased, however, root growth showed a significant reduction. Maturity group (MG) III had significantly lower values for the measured root, shoot, and physiological traits across temperature treatments when compared with MG IV and V. Cultivar variability existed and reflected considerably through positive or negative responses in growth to LT and HT. Cumulative stress response indices and principal component analysis were used to identify cultivar-specific tolerance to temperatures. Based on the analysis, cultivars CZ 5225 LL and GS47R216 were identified as most sensitive and tolerant to LT, while, cultivars 45A-46 and 5115LL identified as most tolerant and sensitive to HT, respectively. The information on cultivar-specific tolerance to low or high temperatures obtained in this study would help in cultivar selection to minimize stand loss in present production areas.
\end{abstract}

Keywords: cumulative stress response indices; conventional soybean production system; early soybean production system; maturity groups; root morphology

\section{Introduction}

Soybean is an important oilseed crop in the US Midsouth, where an average air temperature of $30{ }^{\circ} \mathrm{C}$ is considered ideal for germination and seedling emergence [1]. However, soybean planting dates vary from early March to late May depending upon the type of production system followed, namely, early soybean production system (ESPS) and conventional soybean production system (CSPS) [2,3]. The CSPS involves May and later plantings of soybean varieties belonging to maturity group (MG) 
V-VIII, which allows rapid seed germination and emergence [4]. Whereas ESPS involves planting early-maturing varieties, MG III and IV, from late-March to early-April [5]. Soybean acres and yields are consistently increased in the US Midsouth since the shift from CSPS to ESPS, which provides benefits of early season rainfall, avoids reproduction stage from mid-summer drought and high temperatures, prevents late-season insect-attack, and potential early harvest [1]. However, farmers may risk the exposure of early-growth (seed germination and seedling emergence) of soybean to chilling injury under ESPS, leading to uneven and poor stand establishment [5]. Thus, planting too early under EPSP and too late under CSPS could expose soybean seedling growth to both low- and high-temperatures, respectively, in the US Midsouth.

During the early germination process of soybean, low temperatures can significantly reduce the rate of imbibition, the ability of embryo tissue to expand, and mitochondrial respiration $[6,7]$. Further, susceptibility to chilling injury increases with decreasing initial moisture content in the embryo [6]. The rate of hypocotyl elongation significantly decreases with decreasing temperature below $30{ }^{\circ} \mathrm{C}$ [8]. Interestingly, after effects of low temperatures during the seedling stage can substantially extend the vegetative growth rate, and increase number of axillary branches, the rate of dry weight per plant and pod setting [9]. Whereas, the effects of high temperatures are mostly studied and considered damaging on the reproductive growth and yield potential in soybean, especially under the CSPS system $[5,10,11]$. Many argue that the success of the ESPS system was due to continuously increasing global air temperatures over the years $[10,12,13]$ and they emphasize the importance of determining heat/cold tolerance among the available soybean cultivars during early-growth stages. Also, southernmost states of the US with higher spring temperatures are deprived of the ESPS [12].

Currently, numerous soybean varieties are available that are recommended for a given region that may differ in their tolerance to low and high temperatures [1]. Therefore variety selection along with other planting decisions (i.e., planting date, seed rate, and row spacing) is a key to profitable soybean production in a specific environment $[4,14]$. Temperature and photoperiod predominantly affect morphological and physiological growth and development of soybean plant among other environmental variables [15]. While the phenological response to temperature can primarily determine soybean variety selection for cultivation in a given geographical location during early growth-stages with little interaction of photoperiod [15,16], however, photoperiod modifies the response to temperature with changing geographical locations and therefore serves as a basis for classifying the cultivars by maturity group [17]. Studies in the past have determined genotypic variability in phenological responses to temperatures for the traits such as germination, plant height, node number, net photosynthesis, leaf area, and fruit number per plant by either varying the planting date in the field [2,18-21] or utilizing controlled-environment facilities [10,22-26]. However, photoperiod can become a confounding factor when using planting date as a variable to determine the cultivar response to temperature [16]. Therefore, soybean cultivars tolerance to low or high temperatures within or across MGs at constant photoperiods can be best achieved by utilizing controlled-environment facilities.

Also, root architecture is increasingly studied in US Midsouth crops such as rice, corn, and cotton in identifying responses involved in stress tolerance during seedling growth $[22,23,27,28]$, however, little is known about the soybean root system under stressful conditions [3]. Early assessments of whole-root systems without breaking off the finer parts was nearly impractical in the past [29]. For this reason, previous studies mostly screened cultivars for abiotic stress tolerance based on above-growth traits, like height, leaf area, and node numbers $[18,19,29]$. However, the introduction of root phenotype systems, like hydroponics, gels, wax-petroleum layers, and WinRHIZO root scanner, have offered plant and soil scientists to evaluate root system architecture traits with minimal destruction [23,30,31]. Recent studies have successfully exploited the above technologies to define the relationship between temperature stress tolerance and root traits, including root length, diameter, thickness, surface area, and lateral root numbers $[27,28,31]$. Further, differences in correlation between root and shoot traits to different abiotic stresses were also found during the seedling stage [27]. Therefore, combined analysis 
of above- and belowground growth and developmental traits are important in identifying cultivars for abiotic stress tolerance.

The overall objective of this study was to quantify the temperature effects on root and shoot growth of 64 soybean cultivars during the early-growth stage using the sunlit controlled-environment facility. The specific objective was to classify the soybean cultivars for their degree of tolerance to lowand high-temperatures.

\section{Materials and Methods}

This experiment was conducted in Soil-Plant-Atmosphere-Research (SPAR) units, a sunlit controlled-environmental facility located at the Environmental Plant Physiology Laboratory, Mississippi State University, MS, USA during the 2016 growing season [32]. The experiment consisted of a collection of 64 soybean cultivars from maturity groups (MG) III, IV, and V (Table 1) that are most commonly grown in the US Midsouth and were evaluated under three different day/night temperature treatments (TTs) namely, low temperature (LT; $\left.20 / 12{ }^{\circ} \mathrm{C}\right)$, optimum temperature $\left(\mathrm{OT} ; 30 / 22^{\circ} \mathrm{C}\right.$ ), and high temperature $\left(\mathrm{HT} ; 40 / 32^{\circ} \mathrm{C}\right)$. The experiment was organized in completely randomized design with two factorial arrangements (64 cultivars $\times 3$ TTs) replicated three times spatially using nine different SPAR units such that three replications of each treatment combination (cultivar and TT) were represented by three SPAR units. Treated seeds of sixty-four soybean cultivars were sown in 576 polyvinyl chloride (PVC) plastic pots ( $10 \mathrm{~cm}$ diameter and $45.5 \mathrm{~cm}$ tall), each filled with sandy soil and $250 \mathrm{~g}$ of gravel at the bottom. The pots were placed in the SPAR units at the time of sowing. Immediately after sowing, TTs were imposed and continued until harvesting, 20 days after sowing (DAS). Initially, four seeds were seeded in each pot at a depth of $2 \mathrm{~cm}$ and then thinned to 1 plant after emergence. Plants were irrigated three times per day through an automated, computer-controlled drip system with full-strength Hoagland's nutrient solution at 0700, 1200 and $1700 \mathrm{~h}$. All SPAR units were maintained at $400 \mathrm{ppm} \mathrm{CO}_{2}$ throughout the experiment. 
Table 1. Cultivars name, and maturity group of sixty-four soybean cultivars along with temperature, low (LT), optimum (OT), and high (HT), effects on shoot parameters, total plant dry weight, and physiological parameters, measured at 20 days after sowing. The mean value for each parameter related to maturity group (MG) presented below in Italic format.

\begin{tabular}{|c|c|c|c|c|c|c|c|c|c|c|c|c|c|c|c|c|c|c|c|c|c|c|c|c|c|c|}
\hline \multirow[t]{2}{*}{ Company } & \multirow[t]{2}{*}{ Cultivar } & \multirow[t]{2}{*}{ MG } & \multicolumn{3}{|c|}{ Plant Height, cm } & \multicolumn{3}{|c|}{$\begin{array}{l}\text { Mainstem Nodes, } \\
\text { no. } \text { plant }^{-1}\end{array}$} & \multicolumn{3}{|c|}{ Leaf Area, $\mathrm{cm}^{2}$} & \multicolumn{3}{|c|}{ Leaf Weight, $\mathrm{g}$} & \multicolumn{3}{|c|}{ Stem Weight, $\mathrm{g}$} & \multicolumn{3}{|c|}{$\begin{array}{l}\text { Total Plant } \\
\text { Weight, g }\end{array}$} & \multicolumn{3}{|c|}{$\begin{array}{l}\text { Chlorophyll Content } \\
\text { as SPAD Units }\end{array}$} & \multicolumn{3}{|c|}{$\begin{array}{c}\text { Canopy } \\
\text { Temperature, }{ }^{\circ} \mathrm{C}\end{array}$} \\
\hline & & & LT & OT & HT & LT & OT & HT & LT & OT & HT & LT & OT & HT & LT & OT & HT & LT & OT & HT & LT & OT & HT & LT & OT & HT \\
\hline Dyna-Gro Seed & 32 y39 & III & 5 & 13 & 15 & 1 & 3 & 4 & 26 & 206 & 232 & 0.13 & 0.82 & 0.81 & 0.04 & 0.37 & 0.44 & 0.22 & 1.44 & 1.48 & 25 & 37 & 41 & 25 & 30 & 34 \\
\hline Mycogen Seeds & 5N393R2 & III & 4 & 12 & 12 & 1 & 3 & 5 & 46 & 227 & 316 & 0.19 & 0.81 & 1.04 & 0.04 & 0.28 & 0.38 & 0.34 & 1.32 & 1.72 & 29 & 37 & 43 & 25 & 30 & 36 \\
\hline Syngenta United States & S39-T3 & III & 4 & 10 & 11 & 1 & 3 & 5 & & 215 & & 0.11 & 0.83 & 0.89 & 0.03 & 0.31 & & 0.18 & 1.41 & 1.53 & 31 & 40 & 45 & & 29 & 35 \\
\hline Syngenta United States & S39-C4 & III & 4 & 11 & 11 & 1 & 3 & 4 & 29 & 257 & & 0.11 & 1.00 & 97 & 02 & 0.36 & 0.35 & 0.19 & 1.73 & 1.65 & 29 & 40 & 47 & 26 & 31 & 36 \\
\hline \multirow[t]{2}{*}{ REV Brand Seeds } & 38 R10 & III & 4 & 12 & 13 & 1 & 4 & 4 & 43 & 290 & 230 & 0.19 & 1.19 & 0.89 & 0.05 & 0.43 & 0.36 & 0.33 & 2.04 & 1.53 & 28 & 37 & 43 & 25 & 31 & 35 \\
\hline & Mean & III & 4 & 11 & 12 & 1 & 3 & 4 & 34 & 239 & 26 & 0.15 & 0.93 & 0.92 & 0.04 & 0.35 & 0.38 & 0.25 & 1.59 & 1.58 & 28 & 38 & 44 & 25 & 30 & 35 \\
\hline Go Soy Genetics Optimized & IREANE & IV & 5 & 15 & 14 & 1 & 4 & 5 & 40 & 265 & 302 & 0.18 & 0.88 & 1.04 & 0.03 & 0.37 & 0.44 & 0.29 & 1.54 & 1.86 & 24 & 34 & 40 & 24 & 30 & 35 \\
\hline Go Soy Genetics Optimized & $483 . \mathrm{C}$ & IV & 5 & 17 & 18 & 1 & 4 & 5 & 60 & 286 & 266 & 0.32 & 0.96 & 1.16 & 0.06 & 0.43 & 0.48 & 0.49 & 1.78 & 1.90 & 26 & 33 & 42 & 25 & 32 & 35 \\
\hline UniSouth Genetics Inc. & ELLIS & IV & 5 & 13 & 15 & 1 & 4 & 4 & 33 & 237 & 277 & 0.15 & 0.91 & 0.96 & 04 & 0.35 & 0.40 & 0.25 & 1.54 & 1.65 & 26 & 36 & 39 & 25 & 31 & 34 \\
\hline REV & & IV & 4 & 12 & 1 & 1 & 3 & 5 & & & & & & & & & & & & & & & & & & 34 \\
\hline Delta Grow S & DG 4781LL & IV & 5 & 14 & 16 & 1 & 4 & 4 & 38 & 265 & & & 0.93 & 70 & & 0.4 & 0.38 & 0.31 & 1.60 & 1.35 & & & & & & 38 \\
\hline Go Soy Genetics Optimized & 4714LL & IV & 5 & 13 & 16 & 1 & 4 & 4 & 43 & 273 & 251 & 0.15 & 1.04 & 0.86 & 0.04 & 0.43 & 0.41 & 0.29 & 1.79 & 1.61 & 26 & 33 & 39 & 25 & 28 & 34 \\
\hline Progeny Ag Products & P 4247LL & IV & 5 & 11 & 12 & 1 & 3 & 5 & 44 & 284 & 327 & 0.16 & 0.98 & 1.15 & 0.03 & 0.40 & 0.46 & 0.28 & 1.79 & 2.07 & 27 & 37 & 41 & 25 & 30 & 35 \\
\hline Bayer Credenz & CZ 4044 LL & IV & 5 & 13 & 15 & 1 & 4 & 5 & 30 & 257 & 360 & 0.16 & 0.96 & 1.15 & 03 & 0.39 & 0.51 & 0.23 & 1.63 & 2.01 & 27 & 38 & 43 & 25 & 29 & 35 \\
\hline Dyna-Gro Seeds & $\begin{array}{l}\text { S49LL34 } \\
\text { S49 }\end{array}$ & IV & 4 & 14 & 16 & 1 & 3 & 5 & 42 & 245 & 259 & 0.34 & 0.79 & 0.85 & 04 & 0.34 & 0.36 & 0.47 & 1.41 & 1.48 & 26 & 35 & 40 & 25 & 28 & 35 \\
\hline & & IV & 5 & 11 & 10 & 1 & 4 & 4 & & 278 & & & 1.10 & & & & & & & & & & 40 & & & 35 \\
\hline Delta Grow Seeds Com. Inc. & DG 4680RI & IV & 5 & 12 & 13 & 1 & 3 & 4 & 40 & & & & & & & & & & & & & & & & & 35 \\
\hline REV Brand Seeds & $45 \mathrm{~A} 46$ & IV & 6 & 12 & 15 & 1 & 3 & 5 & 29 & 174 & 340 & 0.14 & 0.63 & 1.23 & 0.04 & 0.24 & 0.49 & 0.25 & 1.09 & 2.05 & 24 & 35 & 43 & & 29 & 33 \\
\hline Mycogen Seeds & $5 \mathrm{~N} 424 \mathrm{R} 2$ & IV & 5 & 11 & 11 & 1 & 4 & 4 & 28 & 274 & 281 & 0.13 & 0.94 & 0.89 & 0.04 & 0.37 & 0.37 & 0.24 & 1.70 & 1.58 & 28 & 37 & 41 & 25 & 31 & 35 \\
\hline Dyna-Gro Seed & $31 \mathrm{RY} 45$ & IV & 4 & 11 & 12 & 1 & 4 & 4 & 27 & 282 & 285 & 0.15 & 1.18 & 1.03 & 04 & 0.48 & 0.48 & 0.27 & 2.10 & 1.85 & 28 & 36 & 44 & 24 & 30 & 34 \\
\hline AGSouth Genetics & GS45R216 & IV & 5 & 13 & 14 & 1 & 4 & 4 & 41 & 242 & 248 & 0.24 & 0.85 & 0 & 02 & 0.3 & & 0.35 & 1.53 & 1.49 & & & 42 & & 30 & 36 \\
\hline Asgrow & & IV & 4 & 12 & 13 & 1 & $\begin{array}{l}4 \\
4\end{array}$ & 4 & & & & & & & & & & & & & & & & & & 34 \\
\hline Prog & & & 5 & 14 & & 1 & 4 & 4 & & & & & & & & & & & & & & & & & & 36 \\
\hline & & IV & 4 & 10 & 1 & 1 & 3 & 4 & & & & & & & & & & & & & & & & & & 33 \\
\hline Bayer Credenz & CZ 4181 RY & IV & 5 & 11 & 12 & 1 & 3 & 4 & 40 & 238 & 350 & 0.21 & 0.80 & 1.32 & 04 & 0.36 & 0.53 & 0.31 & $\begin{array}{l}1.20 \\
1.42\end{array}$ & 2.27 & 26 & 36 & 42 & 26 & 31 & 34 \\
\hline Delta Grow Seed Com. Inc. & DG 825RR2/STS & IV & 5 & 12 & 12 & 1 & 4 & 4 & 46 & 278 & & & 1.1 & & 06 & & & 0.41 & & 1.54 & 29 & 40 & 45 & 24 & 27 & 36 \\
\hline DuPc & & IV & 5 & 12 & 11 & 1 & 3 & 4 & 37 & 192 & & & & & & & & 21 & & & 28 & 36 & 41 & 25 & 29 & 36 \\
\hline Syngenta & & IV & 5 & 11 & 10 & 1 & 3 & 4 & 3 & 234 & & & & & & & & & & & 26 & & 44 & & 29 & 35 \\
\hline AGSo & & & 6 & 15 & & & 4 & 5 & & & & & & & & & & & & & & & & & & 34 \\
\hline & $-\mathrm{R}$ & & & 1 & & & & & & & & & & & & & & & & & & & & & & 34 \\
\hline & & IV & & 1 & 1 & & & 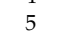 & & & & & & & & & & & & & & & & & & 35 \\
\hline REV B & & IV & 5 & 13 & 1 & 1 & 4 & 5 & & & & & & & & & & & & & 2 & 36 & 44 & & 30 & 36 \\
\hline & & IV & 6 & 12 & 13 & 1 & 3 & 4 & & & & & & & & & & 23 & & & 28 & 37 & 40 & 24 & 29 & 36 \\
\hline & & IV & 5 & 15 & 13 & & 4 & 4 & & & & & & & & & & & & & 31 & & 46 & & 30 & 35 \\
\hline Go Soy G & 4 & IV & 4 & 11 & 11 & & 4 & $\begin{array}{l}4 \\
4\end{array}$ & & & & & & & & & & & 44 & & & & 42 & & 30 & 35 \\
\hline Croplan & & & 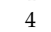 & 11 & & & 3 & 4 & & & & & & & & & & & & & & & & & & 35 \\
\hline & & $\mathrm{I}$ & & & & & & & & & & & & & & & & & & & & & & & & \\
\hline & & I & 5 & 15 & 14 & 1 & 4 & 4 & & & & & & & & & & & & & & & 44 & & & 35 \\
\hline & & I & 4 & 12 & 12 & 1 & 4 & 4 & & & & & & & & & & & & & 29 & 2 & 41 & & 31 & 37 \\
\hline State Universit & & IV & 4 & 15 & 15 & 1 & 4 & 5 & 38 & 364 & 330 & 0.24 & 1.25 & & 04 & & & 0.34 & 2.14 & 1.96 & 29 & 40 & 42 & 25 & 30 & 32 \\
\hline University of Missouri & R01-416F & IV & 5 & 12 & 12 & 1 & 4 & 4 & & 287 & 254 & 0.19 & 1.17 & & 0.05 & 0.47 & & 0.30 & 2.01 & 1.60 & 26 & 41 & 45 & & 31 & 35 \\
\hline & Mean & IV & 5 & 13 & 13 & 1 & 4 & 4 & & & & & & & & & & & 1.73 & & 27 & & 42 & & 30 & 35 \\
\hline & & $\mathrm{V}$ & & 11 & 11 & & & 1 & & & & & & & & & & & & & & & & & & \\
\hline & & $\mathrm{V}$ & & 1 & 1 & & & & & & & & & & & & & & & & & & & & & \\
\hline USDA-ARS & JTN-5110 & $\mathrm{V}$ & 4 & 12 & 12 & 1 & 4 & 4 & 35 & 352 & 337 & 0.17 & 1.57 & 1.26 & 0.05 & 0.66 & 0.43 & 0.30 & 2.69 & 2.09 & 27 & 38 & 42 & 25 & 29 & 34 \\
\hline
\end{tabular}


Table 1. Cont.

\begin{tabular}{|c|c|c|c|c|c|c|c|c|c|c|c|c|c|c|c|c|c|c|c|c|c|c|c|c|c|c|}
\hline \multirow[t]{2}{*}{ Company } & \multirow[t]{2}{*}{ Cultivar } & \multirow[t]{2}{*}{ MG } & \multicolumn{3}{|c|}{ Plant Height, cm } & \multicolumn{3}{|c|}{$\begin{array}{l}\text { Mainstem Nodes, } \\
\text { no. } \text { plant }^{-1}\end{array}$} & \multicolumn{3}{|c|}{ Leaf Area, $\mathrm{cm}^{2}$} & \multicolumn{3}{|c|}{ Leaf Weight, $\mathrm{g}$} & \multicolumn{3}{|c|}{ Stem Weight, $\mathrm{g}$} & \multicolumn{3}{|c|}{$\begin{array}{l}\text { Total Plant } \\
\text { Weight, g }\end{array}$} & \multicolumn{3}{|c|}{$\begin{array}{l}\text { Chlorophyll Content } \\
\text { as SPAD Units }\end{array}$} & \multicolumn{3}{|c|}{$\begin{array}{c}\text { Canopy } \\
\text { Temperature, }{ }^{\circ} \mathrm{C}\end{array}$} \\
\hline & & & LT & OT & HT & LT & OT & HT & LT & OT & HT & LT & OT & HT & LT & OT & HT & LT & OT & HT & LT & OT & HT & LT & OT & HT \\
\hline Go Soy Genetics Optimized & LELAND & $\mathrm{V}$ & 5 & 14 & 14 & 1 & 4 & 4 & 32 & 283 & 258 & 0.17 & 1.02 & 0.88 & 0.02 & 0.39 & 0.38 & 0.26 & 1.69 & 1.53 & 24 & 38 & 44 & 25 & 30 & 34 \\
\hline Delta Grow Seeds Co Inc & DG5067LL & $\mathrm{V}$ & 4 & 11 & 13 & 1 & 3 & 5 & 40 & 225 & 311 & 0.20 & 0.96 & 1.09 & 0.05 & 0.31 & 0.44 & 0.35 & 1.57 & 1.84 & 28 & 38 & 45 & 25 & 30 & 35 \\
\hline Go Soy Genetics Optimized & 5115LL & $\mathrm{V}$ & 5 & 11 & 12 & 1 & 3 & 4 & 49 & 276 & 209 & 0.26 & 1.01 & 0.70 & 0.05 & 0.37 & 0.30 & 0.40 & 1.77 & 1.19 & 26 & 35 & 39 & 25 & 29 & 36 \\
\hline Dyna-Gro Seed & S55LS75 & $\mathrm{V}$ & 5 & 14 & 15 & 1 & 3 & 5 & 34 & 248 & 282 & 0.16 & 1.03 & 0.87 & 0.03 & 0.47 & 0.48 & 0.25 & 1.78 & 1.71 & 25 & 35 & 39 & 24 & 29 & 35 \\
\hline Bayer Credenz & CZ 5242 LL & $\mathrm{V}$ & 5 & 11 & 12 & 1 & 3 & 4 & 34 & 200 & 287 & 0.15 & 0.65 & 0.89 & 0.03 & 0.28 & 0.40 & 0.24 & 1.13 & 1.57 & 26 & 35 & 41 & 25 & 30 & 36 \\
\hline Bayer Credenz & CZ 5225 LL & $\mathrm{V}$ & 5 & 15 & 15 & 1 & 4 & 4 & 27 & 218 & 269 & 0.15 & 0.78 & 0.82 & 0.03 & 0.39 & 0.42 & 0.22 & 1.41 & 1.50 & 28 & 36 & 40 & 26 & 30 & 35 \\
\hline Delta Grow Seeds Com. Inc. & DG 5170RR2/STS & $\mathrm{V}$ & 5 & 11 & 13 & 1 & 4 & 4 & 38 & 339 & 299 & 0.19 & 1.33 & 1.09 & 0.04 & 0.46 & 0.44 & 0.30 & 2.14 & 1.87 & 29 & 38 & 43 & 25 & 29 & 34 \\
\hline $\begin{array}{l}\text { REV Brand Seeds } \\
\text { S }\end{array}$ & $51 \mathrm{~A} 56$ & $\mathrm{~V}$ & 5 & 12 & 13 & 1 & 4 & 4 & 47 & 239 & 280 & 0.20 & 0.96 & 0.97 & 0.05 & 0.35 & 0.46 & 0.32 & 1.60 & 1.69 & 27 & 35 & 38 & 25 & 29 & 34 \\
\hline DuPont Pioneer & P52T50R & $\mathrm{V}$ & 4 & 13 & 13 & 1 & 4 & 4 & 39 & 290 & 276 & 0.12 & 1.12 & 1.03 & 0.03 & 0.41 & 0.44 & 0.23 & 1.84 & 1.78 & 24 & 37 & 43 & 25 & 30 & 35 \\
\hline Syngenta United States & S55-Q3 & $\mathrm{V}$ & 5 & 14 & 15 & 1 & 4 & 4 & 47 & 231 & 273 & 0.18 & 0.77 & 0.89 & 0.07 & 0.30 & 0.36 & 0.32 & 1.30 & 1.54 & 26 & 37 & 44 & 25 & 30 & 37 \\
\hline Syngenta United States & S56-M8 & $\mathrm{V}$ & 5 & 14 & 13 & 1 & 3 & 4 & 39 & 198 & 201 & 0.10 & 0.66 & 0.66 & 0.03 & 0.19 & 0.26 & 0.17 & 1.05 & 1.15 & 24 & 36 & 44 & 25 & 29 & 36 \\
\hline Go Soy Genetics Optimized & 5214GTS & $\mathrm{V}$ & 5 & 17 & 15 & 1 & 4 & 5 & 37 & 315 & 365 & 0.15 & 1.18 & 1.14 & 0.03 & 0.54 & 0.52 & 0.24 & 2.04 & 1.98 & 27 & 36 & 45 & 23 & 28 & 35 \\
\hline Armor & 55-R68 & $\mathrm{V}$ & 4 & 14 & 13 & 1 & 4 & 4 & 36 & 307 & 330 & 0.17 & 1.18 & 0.95 & 0.05 & 0.47 & 0.44 & 0.28 & 1.99 & 1.70 & 24 & 33 & 39 & 24 & 31 & 36 \\
\hline Progeny Ag Products & P 5226RYS & $\mathrm{V}$ & 4 & 12 & 13 & 1 & 4 & 5 & 45 & 322 & 280 & 0.25 & 1.07 & 0.98 & 0.03 & 0.42 & 0.40 & 0.37 & 1.84 & 1.63 & 26 & 36 & 42 & 25 & 30 & 35 \\
\hline Mycogen Seeds & 5N523R2 & $\mathrm{V}$ & 4 & 12 & 12 & 1 & 3 & 4 & 32 & 277 & 231 & 0.17 & 1.03 & 0.92 & 02 & 0.39 & 0. & 0.28 & 1.77 & 1.59 & 29 & 38 & 42 & 25 & 29 & 35 \\
\hline Dyna-Gro seed & S56RY84 & $\mathrm{v}$ & 5 & 16 & 15 & 1 & 4 & 5 & 51 & 306 & 322 & 0.25 & 1.04 & 1.02 & 0.05 & 0.51 & 0.48 & 0.41 & 1.89 & 1.87 & 24 & 35 & 38 & 25 & 29 & 35 \\
\hline Croplan WinField United & R2C5225S & $\mathrm{V}$ & 4 & 12 & 12 & 1 & 3 & 5 & 41 & 335 & 282 & 0.26 & 1.16 & 1.03 & 0.03 & 0.51 & 0.46 & 0.38 & 2.04 & 1.84 & 27 & 37 & 40 & 25 & 29 & 33 \\
\hline Bayer Credenz & CZ 5375 RY & $\mathrm{V}$ & 5 & 11 & 12 & 1 & 3 & 4 & 34 & 244 & 330 & 0.18 & 0.87 & 1.07 & 0.04 & 0.30 & 0.43 & 0.28 & 1.45 & 1.87 & 26 & 35 & 42 & 25 & 31 & 36 \\
\hline REV Brand Seeds & $57 \mathrm{R} 21$ & $\mathrm{~V}$ & 5 & 15 & 13 & 1 & 4 & 4 & 46 & 293 & 278 & 0.21 & 1.03 & 1.08 & 0.04 & 0.46 & 0.36 & 0.30 & 1.80 & 1.77 & 26 & 34 & 46 & 25 & 30 & 35 \\
\hline Syngenta United States & S58-Z4 & $\mathrm{V}$ & 5 & 12 & 12 & 1 & 3 & 4 & 28 & 203 & 247 & 0.17 & 0.70 & 0.81 & 0.03 & 0.28 & 0.36 & 0.24 & 1.19 & 1.45 & 28 & 34 & 40 & 25 & 28 & 35 \\
\hline Dyna-Gro Seed & $\begin{array}{l}\mathrm{S} 50-24 \\
\text { S57RY26 }\end{array}$ & $\mathrm{V}$ & 6 & 14 & 15 & 1 & 4 & 4 & 43 & 303 & 350 & 0.22 & 1.12 & 1.20 & 0.03 & 0.47 & 0.47 & 0.35 & $\begin{array}{l}1.94 \\
1.94\end{array}$ & 2.02 & 27 & 36 & 45 & 25 & 31 & 34 \\
\hline & Mean & V & & 13 & 13 & 1 & 4 & 4 & 39 & 274 & 287 & 0.18 & 1.01 & 0.98 & 0.04 & 0.41 & 0.42 & 0.30 & 1.73 & 1.70 & 26 & 36 & 42 & 25 & 30 & 35 \\
\hline $\begin{array}{c}\text { Mean } \\
+ \text { ANOVA }\end{array}$ & & & 5 & 13 & 13 & 1 & 4 & 4 & 37 & 267 & 273 & 0.18 & 1.00 & 0.95 & 0.04 & 0.40 & 0.41 & 0.29 & 1.72 & 1.66 & 27 & 36 & 42 & 25 & 30 & 35 \\
\hline MG & & & & $\stackrel{t}{+}$ & & & ns & & & $* * *$ & & & ns & & & ns & & & ns & & & $* * *$ & & & ns & \\
\hline CUL & & & tns & $* * *$ & $* * *$ & ns & ns & ns & $* * *$ & $* * *$ & $* * *$ & $* * *$ & $* * *$ & ns & ns & $* * *$ & ns & $* * *$ & $* * *$ & ns & **** & $* * *$ & $* * *$ & ns & ns & ns \\
\hline TT & & & & $* * *$ & & & $* * *$ & & & $* * *$ & & & $* * *$ & & & $* * *$ & & & $* * *$ & & & $* * *$ & & & **** & \\
\hline LT & & & & $* * *$ & & & $* * *$ & & & ns & & & $* * *$ & & & ns & & & ns & & & $* * *$ & & & $* * *$ & \\
\hline HT & & & & $* * *$ & & & $* * *$ & & & $* * *$ & & & $* * *$ & & & $* * *$ & & & $* * *$ & & & $* * *$ & & & $* * *$ & \\
\hline $\mathrm{MG} \times \mathrm{TT}$ & & & & ns & & & ns & & & $* * *$ & & & ns & & & ns & & & ns & & & $* * *$ & & & ns & \\
\hline $\mathrm{CUL} \times \mathrm{TT}$ & & & & $* * * *$ & & & ns & & & $* * *$ & & & $* * *$ & & & $t^{*}$ & & & $* * * *$ & & & ns & & & ns & \\
\hline
\end{tabular}

$t^{*}, * * *$ and ns representing significance at the $p \leq 0.05, p<0.001$, and non-significant $(p>0.05)$, respectively. CUL, cultivar; MG, maturity group; TT, temperature treatment; LT, low-temperature treatment; HT, high-temperature treatment; OT, optimum temperature treatment. 


\subsection{Measurements}

Physiological parameters such as chlorophyll content were measured using chlorophyll estimates measured and presented as Soil-Plant-Analysis-Development (SPAD) units (SPAD-502, Minolta Camera Co. Ltd., Osaka, Japan) and canopy temperature using an infrared thermometer (MI-230, Apogee Instruments, Inc., Logan, UT, USA) were measured on the day before the harvest between 10:00 to 12:00 a.m. Shoot parameters such as plant height $(\mathrm{PH})$, mainstem node number $(\mathrm{NN})$, and leaf area (LA) using leaf area meter (Li-3100, Li-COR Inc., Lincoln, Nebraska, USA) were measured at the time of harvest. Root parameters such as cumulative root length (CRL), root surface area (RSA), root diameter (RD), lateral root numbers (i.e., numbers of root tips (RT), forks (RF), crossings (RC)), and root volume (RV) were measured and analyzed using the Win-RHIZO optical scanner according to the methods described previously $[27,28,30,31]$. After that, plant-component dry weights, stems, leaves, and roots, were obtained by oven-drying at $80^{\circ} \mathrm{C}$, and root/shoot ratio was calculated accordingly.

\subsection{Cumulative Stress Response Indices}

Cumulative stress response indices for LT (CLTRI) and HT (CHTRI) were calculated to classify soybean cultivars based on their degree of tolerance to LT and HT, respectively. Koti et al. [33] defined cumulative stress response index (CSRI) as the sum of relative individual component responses under each treatment. Accordingly, individual stress response indices for LT (ILTRI) and HT (IHTRI) for each cultivar were obtained by dividing the value of parameter obtained at LT or HT by the value of the same parameter obtained at OT. The calculations were done for all measured parameters. Then, CLTRI and CHTRI were calculated for each cultivar by summing ILTRI and IHTRI, respectively. Finally, soybean cultivars were classified as sensitive, moderately sensitive, moderately tolerant, and highly tolerant to LT or HT based on CLTRI or CHTRI values, and an increment of one standard deviation, respectively, as described by Koti et al. [33].

\subsection{Data Analysis}

Considering all SPAR chambers have the same growth conditions, except temperature, the assignment of temperature treatments to a given SPAR unit was randomized and cultivars were completely randomized within each unit, therefore, the study was treated as a completely randomized design for statistical analysis purposes. Proc ANOVA analysis procedure (ANOVA) was performed on the replicated values of the measured parameters using PROC GLM procedure in SAS (SAS Institute, Inc., Cary, NC, USA) to determine the effect of cultivar, TT, MG, and their interaction. Post ANOVA means comparison was made using least significant difference $(L S D=0.05)$. Pearson's correlation coefficients for pairs of shoot, root, and physiological traits were calculated at $\alpha$ level of $5 \%$. Sigma plot 13.0 (Systat Software, Inc., San Jose, CA, USA) was used to generate graphs and correlations using best-fitted regression functions.

\subsection{Principal Component Analysis (PCA)}

The principal component analysis was performed to identify the parameters that best describe either low or high-temperature tolerance to response variables and to classify cultivars into different temperature tolerant groups. The analysis was conducted with the PRINCOMP procedure of SAS (SAS Institute, Inc., Cary, NC, USA) and the results were summarized in biplots using SigmaPlot 13 (Systat Software, Inc., San Jose, CA, USA), which are the plots of the mean principal component scores (PC scores) for the treatments of first two principal components. PCA was performed on the correlation matrix of 64 soybean cultivars and 16 response variables comprising plant height $(\mathrm{PH})$, mainstem nodes number (NN), leaf area (LA), stem weight (SW), leaf weight (LW), root weight (RW), total weight (TW), root length (RL), root surface area (RSA), root average diameter (RAD), root volume (RV), canopy temperature (CT), root tips (RNT), root forks (RNF), root crossings (RC), and root-shoot ratio (RS). The superimposed biplot was developed by plotting eigenvectors for the 16 responses as 
solid circles and cultivars as open stars projecting from the origin into various positions. The values of eigenvectors and PC scores were used to classify soybean cultivars into LT and HT tolerant groups.

\section{Results}

\subsection{Growth and Development}

\subsubsection{Shoot Parameters}

The study revealed significant cultivar, TT, MG, and their interaction effects on most of the measured parameters (Tables 1 and 2). Among shoot parameters, TTs significantly affected $\mathrm{PH}$, $\mathrm{NN}$, and LA $(p<0.001)$. On an average across cultivars, the values for PH and NN significantly increased $(p<0.001)$ with increasing temperatures from low to high, but LA was significantly reduced under LT effects with no differences were observed between OT and HT ( $p>0.05)$. Maturity groups significantly differed for PH and LA $(p<0.001)$, while MG $\times$ TT interactions were only significant for LA (Table 1). However, cultivars showed significant variability for PH and LA $(p<0.001)$ under the TTs, except under LT effects (Table 1 ). Further, no variations among the cultivars were observed for $\mathrm{NN}(p>0.05)$. Also, interaction effect (cultivar $\times$ TT) was significant for PH and LA $(p<0.001)$, but not for NN. The averaged PH increased from 5 to $13 \mathrm{~cm}$, LA from 1 to $4 \mathrm{~cm}^{2}$, and NN from 37 to 273, respectively, when compared between OT and HT. Cultivars 4714, 48L63, and 45A46 showed a greater increase in $\mathrm{PH}$ among other cultivars with increasing temperatures from optimum to high (Table 1).

In contrast, cultivars P41T33R, P 4588RY, and AR4705 grown at OT were taller than at HT. The maximum and minimum values for $\mathrm{PH}$ were observed in cultivar $483 \mathrm{C}$ at $\mathrm{HT}$ and cultivar 55-R68 at LT, respectively. Overall, cultivars belonging to MG III had significantly lower values for PH (Figure 1A), while differences were not significant between MG IV and V (Table 1). At low temperature, $11 \%$ reduction was observed in plant height for cultivars from MG III when compared to MG IV and V (Figure 1A). The highest and lowest percent increase in LA was observed in cultivar JTN-5110 and P47T36R, respectively, when compared between LT and OT effects (Table 1). Cultivars like CZ 4044 LL, 45A46, and CZ $4181 \mathrm{RY}$ showed greater values for LA with increasing temperatures from optimal to high, while LA of cultivars like S45-W9, and P41T33R was smaller under OT than HT. Similar to plant height, cultivars belonging to MG V, showed 9 and $12 \%$ increase in leaf area at high and low temperature compared to the cultivars from MG III (Figure 1B). Moreover, some cultivars like S47-K5, S48RS53, R01-416F, and JTN-5110 showed lower values for both PH as well as LA under HT than OT (Table 1). Cultivar 5214GTS at HT showed highest, and cultivar S39-T3 at LT showed the lowest value for LA, respectively. On an average across TTs, MG V cultivars had greater LA than MG III and IV (Table 1). 
Table 2. Cultivars name, and maturity group of sixty-four soybean cultivars along with temperature, low (LT), optimum (OT), and high (HT), effects on root growth and development traits, measured at 20 days after sowing. The mean value for each parameter related to maturity group (MG) presented below in Italic format.

\begin{tabular}{|c|c|c|c|c|c|c|c|c|c|c|c|c|c|c|c|c|c|c|c|c|c|c|c|c|c|c|c|c|c|}
\hline \multirow{2}{*}{ Company } & \multirow[t]{2}{*}{ Cultivar } & \multirow[t]{2}{*}{ MG } & \multicolumn{3}{|c|}{ CRL, cm plant ${ }^{-1}$} & \multicolumn{3}{|c|}{ RSA, $\mathrm{cm}^{2}$ plant $^{-1}$} & \multicolumn{3}{|c|}{ RAD, $\mathrm{cm}$} & \multicolumn{3}{|c|}{$\mathrm{RV}, \mathrm{cm}^{3}$ plant $^{-1}$} & \multicolumn{3}{|c|}{ RNT, no. plant ${ }^{-1}$} & \multicolumn{3}{|c|}{ RNF, no. plant ${ }^{-1}$} & \multicolumn{3}{|c|}{ RNC, no. plant ${ }^{-1}$} & \multicolumn{3}{|c|}{$R / S$} & \multicolumn{3}{|c|}{$\mathrm{RW}, \mathrm{g}$ plant $^{-1}$} \\
\hline & & & LT & OT & HT & LT & OT & HT & LT & OT & HT & LT & OT & HT & LT & OT & HT & LT & OT & HT & LT & OT & HT & LT & OT & HT & LT & OT & HT \\
\hline Dyna-Gro Seed & $32 y 39$ & III & 414 & 2251 & 1987 & 72 & 312 & 274 & 0.5 & 0.4 & 0.4 & 1.0 & 3.4 & 3.0 & 2189 & 3703 & 4220 & 1141 & 6706 & 6570 & 80 & 830 & 781 & 1.23 & 0.66 & 0.54 & 0.1 & 0.2 & 0.2 \\
\hline Mycogen Seeds & 5N393R2 & III & 717 & 2026 & 2389 & 125 & 296 & 346 & 0.6 & 0.5 & 0.5 & 1.8 & 3.5 & 4.0 & 1371 & 4021 & 4998 & 1332 & 5871 & 8860 & 165 & 688 & 1082 & 2.58 & 0.84 & 0.80 & 0.1 & 0.2 & 0.3 \\
\hline Syngenta United States & $\begin{array}{l}\text { S39-T3 } \\
\end{array}$ & III & 372 & 2340 & 2213 & 67 & 337 & 302 & 0.6 & 0.5 & 0.4 & 1.0 & 3.9 & 3.3 & 2028 & 6284 & 4782 & 659 & 7544 & 8507 & 65 & 801 & 1049 & 1.10 & 0.90 & 0.79 & 0.0 & 0.3 & 0.3 \\
\hline Syngenta United States & S39-C4 & III & 560 & 2609 & 2407 & 98 & 403 & 341 & 0.6 & 0.5 & 0.5 & 1.4 & 5.0 & 3.8 & 1571 & 5994 & 5323 & 1108 & 9101 & 9813 & 126 & 966 & 1154 & 2.43 & 1.02 & 0.97 & 0.1 & 0.4 & 0.3 \\
\hline REV Brand Seeds & 38 R10 & III & 743 & 3092 & 2384 & 117 & 465 & 333 & 0.5 & 0.5 & 0.4 & 1.5 & 5.6 & 3.7 & 1621 & 3768 & 4687 & 1485 & 11,861 & 9018 & 200 & 1373 & 1070 & 1.80 & 0.95 & 0.79 & 0.1 & 0.4 & 0.3 \\
\hline & Mean & III & 561 & 2464 & 2276 & 96 & 363 & 319 & 0.6 & 0.5 & 0.4 & 1.3 & 4.3 & 3.6 & 1756 & 4754 & 4802 & 1145 & 8217 & 8554 & 127 & 932 & 1027 & 1.8 & 0.9 & 0.8 & 0.1 & 0.3 & 0.3 \\
\hline Go Soy Genetics Optimized & IREANE & IV & 644 & 2752 & 3090 & 102 & 356 & 435 & 0.5 & 0.4 & 0.4 & 1.3 & 3.7 & 4.9 & 1367 & 7271 & 7120 & 1414 & 9787 & 15,723 & 162 & 1189 & 1659 & 2.78 & 0.80 & 0.86 & 0.1 & 0.3 & 0.4 \\
\hline Go Soy Genetics Optimized & $483 . \mathrm{C}$ & IV & 998 & 3558 & 2359 & 156 & 488 & 320 & 0.5 & 0.4 & 0.4 & 2.0 & 5.3 & 3.5 & 2756 & 5599 & 5694 & 2717 & 15,255 & 9192 & 290 & 1908 & 961 & 2.00 & 0.91 & 0.53 & 0.1 & 0.4 & 0.3 \\
\hline UniSouth Genetics Inc. & ELLIS & IV & 679 & 2115 & 2938 & 100 & 277 & 382 & 0.5 & 0.4 & 0.4 & 1.2 & 3.0 & 4.0 & 1320 & 4358 & 5918 & 1229 & 7234 & 13,986 & 215 & 861 & 1641 & 1.64 & 0.78 & 0.73 & 0.1 & 0.3 & 0.3 \\
\hline REV Brand Seeds & $48 \mathrm{~L} 63$ & IV & 502 & 2188 & 2475 & 82 & 324 & 350 & 0.5 & 0.5 & 0.4 & 1.1 & 3.8 & 3.9 & 1405 & 3446 & 5861 & 918 & 7742 & 8929 & 107 & 846 & 1005 & 1.55 & 0.74 & 0.66 & 0.1 & 0.3 & 0.3 \\
\hline Delta Grow Seeds Com. Inc. & DG 4781LL & IV & 754 & 2393 & 2606 & 133 & 331 & 360 & 0.6 & 0.4 & 0.4 & 1.9 & 3.6 & 4.0 & 1871 & 9450 & 6631 & 1379 & 7623 & 9744 & 180 & 814 & 1123 & 1.86 & 0.68 & 0.70 & 0.1 & 0.3 & 0.3 \\
\hline Go Soy Genetics Optimized & $4714 \mathrm{LL}$ & IV & 742 & 2691 & 2565 & 122 & 390 & 362 & 0.5 & 0.5 & 0.5 & 1.7 & 4.5 & 4.1 & 2458 & 5725 & 5700 & 1667 & 9090 & 11,462 & 165 & 1010 & 1116 & 2.33 & 0.74 & 0.82 & 0.1 & 0.3 & 0.3 \\
\hline Progeny Ag Products & P 4247LL & IV & 714 & 3333 & 3337 & 120 & 491 & 486 & 0.5 & 0.5 & 0.5 & 1.7 & 5.8 & 6.0 & 1346 & 5959 & 6628 & 1748 & 12,758 & 14,741 & 222 & 1406 & 1459 & 3.11 & 1.01 & 1.01 & 0.1 & 0.4 & 0.5 \\
\hline Bayer Credenz & CZ 4044 LL & IV & 510 & 2873 & 2629 & 78 & 394 & 346 & 0.5 & 0.4 & 0.4 & 1.0 & 4.3 & 3.6 & 1481 & 4804 & 5072 & 1048 & 9815 & 10,136 & 135 & 1205 & 1321 & 1.33 & 0.73 & 0.69 & 0.0 & 0.3 & 0.4 \\
\hline Dyna-Gro Seeds & S49LL34 & IV & 555 & 2238 & 2553 & 94 & 330 & 355 & 0.5 & 0.5 & 0.4 & 1.3 & 3.9 & 3.9 & 2240 & 3097 & 6125 & 1083 & 7387 & 9296 & 121 & 868 & 1053 & 2.55 & 0.82 & 0.74 & 0.1 & 0.3 & 0.3 \\
\hline DuPont Pioneer & P41T33R & IV & 659 & 3186 & 2751 & 106 & 417 & 391 & 0.5 & 0.4 & 0.5 & 1.4 & 4.4 & 4.4 & 1407 & 5592 & 4015 & 1220 & 11,865 & 11,868 & 142 & 1598 & 1288 & 3.14 & 0.85 & 0.99 & 0.1 & 0.3 & 0.3 \\
\hline Delta Grow Seeds Co Inc & DG 4680RR2 & IV & 639 & 2659 & 2361 & 102 & 380 & 306 & 0.5 & 0.5 & 0.4 & 1.3 & 4.3 & 3.2 & 1939 & 6207 & 5670 & 1586 & 9585 & 9512 & 188 & 1069 & 1150 & 2.63 & 0.86 & 0.77 & 0.1 & 0.4 & 0.3 \\
\hline REV Brand Seeds & $45 \mathrm{~A} 46$ & IV & 492 & 1887 & 2951 & 82 & 275 & 406 & 0.5 & 0.5 & 0.4 & 1.1 & 3.2 & 4.4 & 2478 & 4987 & 6708 & 1472 & 6456 & 12,079 & 113 & 593 & 1408 & 1.83 & 0.92 & 0.66 & 0.1 & 0.2 & 0.3 \\
\hline Mycogen Seeds & $5 \mathrm{~N} 424 \mathrm{R} 2$ & IV & 642 & 3141 & 2335 & 99 & 420 & 327 & 0.5 & 0.4 & 0.4 & 1.2 & 4.5 & 3.6 & 1558 & 6653 & 5363 & 1282 & 10,741 & 9416 & 183 & 1396 & 1057 & 1.83 & 1.07 & 0.89 & 0.1 & 0.4 & 0.3 \\
\hline Dyna-Gro Seed & $31 \mathrm{RY} 45$ & IV & 597 & 3474 & 3067 & 105 & 509 & 423 & 0.6 & 0.5 & 0.4 & 1.5 & 5.9 & 4.7 & 1553 & 7162 & 8533 & 1036 & 13,543 & 13,046 & 115 & 1541 & 1454 & 2.00 & 0.89 & 0.73 & 0.1 & 0.4 & 0.4 \\
\hline AGSouth Genetics & GS45R216 & IV & 753 & 2895 & 2007 & 124 & 409 & 261 & 0.5 & 0.5 & 0.4 & 1.6 & 4.6 & 2.7 & 1483 & 6759 & 4221 & 2023 & 9841 & 8267 & 209 & 1126 & 1024 & 4.00 & 0.90 & 0.74 & 0.1 & 0.3 & 0.3 \\
\hline Asgrow & 34632 & IV & 564 & 2936 & 2264 & 95 & 440 & 314 & 0.5 & 0.5 & 0.4 & 1.3 & 5.3 & 3.5 & 1546 & 5053 & 4335 & 923 & 11,514 & 9375 & 107 & 1158 & 1044 & 1.90 & 0.95 & 0.66 & 0.1 & 0.4 & 0.3 \\
\hline Progeny Ag Products & P 4588RY & IV & 480 & 2493 & 2273 & 81 & 317 & 309 & 0.5 & 0.4 & 0.4 & 1.1 & 3.2 & 3.3 & 1263 & 9315 & 7881 & 918 & 8437 & 8340 & 100 & 1026 & 930 & 2.00 & 0.73 & 0.92 & 0.1 & 0.3 & 0.2 \\
\hline Syngenta United States & S45-W9 & IV & 393 & 2011 & 1561 & 72 & 281 & 216 & 0.6 & 0.4 & 0.4 & 1.0 & 3.1 & 2.4 & 1191 & 8656 & 4932 & 790 & 5721 & 5300 & 80 & 626 & 592 & 1.63 & 0.95 & 1.05 & 0.0 & 0.3 & 0.2 \\
\hline Bayer Credenz & CZ 4181 RY & IV & 660 & 2221 & 3362 & 99 & 305 & 449 & 0.5 & 0.4 & 0.4 & 1.2 & 3.3 & 4.8 & 1696 & 5495 & 6228 & 1192 & 6991 & 16,099 & 204 & 881 & 1996 & 1.42 & 0.72 & 78 & 0.1 & 0.3 & 0.4 \\
\hline $\begin{array}{l}\text { Delta Grow Seed Com. Inc. } \\
\end{array}$ & G 4825RR2/STS & IV & 710 & 3250 & 2499 & 121 & 447 & 346 & 0.5 & 0.4 & 0.4 & 1.6 & 4.9 & 3.8 & 2894 & 6166 & 4988 & 1613 & 12,772 & 10,034 & 172 & 1513 & 1184 & 1.44 & 0.74 & 0.77 & 0.1 & 0.4 & 0.3 \\
\hline DuPont Pioneer & P47T36R & IV & 631 & 2222 & 1859 & 109 & 311 & 257 & 0.6 & 0.4 & 0.4 & 1.5 & 3.5 & 2.8 & 2377 & 5081 & 4612 & 1530 & 7189 & 6342 & 139 & 845 & 678 & 1.92 & 0.86 & 0.76 & 0.1 & 0.3 & 0.2 \\
\hline Syngenta United States & & IV & 444 & 2700 & 1716 & 69 & 371 & 245 & 0.5 & 0.4 & 0.5 & 0.9 & 4.1 & 2.8 & 1549 & 5477 & 4102 & 949 & 8912 & 5749 & 111 & 1144 & 659 & 1.75 & 0.94 & 96 & 0.0 & 0.3 & 0.2 \\
\hline AGSouth Genetics & GS47R216 & IV & 748 & 2836 & 2521 & 122 & 417 & 352 & 0.5 & 0.5 & 0.4 & 1.6 & 4.9 & 3.9 & 1591 & 5252 & 5759 & 1380 & 10,628 & 9321 & 191 & 1182 & 1170 & 2.23 & 0.76 & 0.81 & 0.1 & 0.4 & 0.4 \\
\hline Armor Seeds & 47-R70 (AR4705) & IV & 672 & 2689 & 2199 & 112 & 348 & 305 & 0.5 & 0.4 & 0.4 & 1.5 & 3.9 & 3.4 & 1640 & 7078 & 5881 & 1141 & 9556 & 8432 & 159 & 1263 & 826 & 2.60 & 0.79 & 0.73 & 0.1 & 0.3 & 0.4 \\
\hline Mycogen Seed & 5N490R2 & IV & 651 & 2207 & 2348 & 101 & 296 & 288 & 0.5 & 0.4 & 0.4 & 1.3 & 3.2 & 2.8 & 2495 & 3797 & 6651 & 1157 & 6614 & 9039 & 137 & 861 & 1157 & 1.62 & 0.70 & 0.68 & 0.1 & 0.3 & 0.3 \\
\hline REV Brand Seeds & $48 \mathrm{~A} 26$ & IV & 591 & 2974 & 2869 & 94 & 424 & 399 & 0.5 & 0.5 & 0.4 & 1.2 & 4.8 & 4.4 & 1790 & 5246 & 6305 & 1259 & 10,112 & 12,486 & 149 & 1232 & 1385 & 1.77 & 0.88 & 0.69 & 0.1 & 0.3 & 0.3 \\
\hline Progeny Ag Products & P 4757RY & IV & 512 & 2676 & 2384 & 77 & 361 & 312 & 0.5 & 0.4 & 0.4 & 0.9 & 3.9 & 3.3 & 2115 & 7402 & 5672 & 1073 & 9354 & 8884 & 112 & 1087 & 1048 & 1.50 & 0.99 & 0.79 & 0.1 & 0.4 & 0.3 \\
\hline Dyna-Gro seeds & S48RS53 & IV & 762 & 2808 & 2013 & 131 & 381 & 289 & 0.6 & 0.4 & 0.4 & 1.8 & 4.1 & 2.6 & 1353 & 3518 & 4320 & 2169 & 10,220 & 7872 & 214 & 1335 & 970 & 2.54 & 0.74 & 0.65 & 0.1 & 0.3 & 0.3 \\
\hline Go Soy Genetics Optimized & $4 \mathrm{GTS}$ & IV & 482 & 2155 & 2413 & 86 & 313 & 306 & 0.6 & 0.5 & 0.4 & 1.2 & 3.6 & 3.1 & 1219 & 5046 & 6238 & 974 & 6611 & 8911 & 98 & 817 & 1194 & 2.44 & 0.85 & 0.77 & 0.1 & 0.3 & 0.2 \\
\hline Croplan WinField United & R2C4775 & IV & 508 & 2769 & 2771 & 82 & 408 & 390 & 0.5 & 0.5 & 0.4 & 1.0 & 4.8 & 4.4 & 3047 & 4911 & 5292 & 1301 & 10,823 & 11,150 & 123 & 1143 & 1339 & 2.57 & 0.82 & 1.03 & 0.1 & 0.3 & 0.4 \\
\hline Bayer Credenz & CZ 4898 RY & IV & 538 & 2256 & 2785 & 88 & 305 & 369 & 0.5 & 0.4 & 0.4 & 1.2 & 3.3 & 3.9 & 2052 & 4975 & 8320 & 1019 & 6800 & 11,555 & 107 & 863 & 1324 & 1.30 & 0.74 & 0.74 & 0.0 & 0.3 & 0.3 \\
\hline Dixie Belle & DB 4911 & IV & 600 & 2828 & 3209 & 83 & 372 & 418 & 0.4 & 0.4 & 0.4 & 0.9 & 3.9 & 4.3 & 1387 & 6270 & 6249 & 1299 & & 15,688 & 213 & 1404 & 68 & 2.83 & 0.85 & 95 & 0.1 & 0.3 & .3 \\
\hline Great Heart Seed Com. & GT-476CR2 & IV & 631 & 2637 & 2310 & 98 & 370 & 265 & 0.5 & 0.4 & 0.4 & 1.2 & 4.2 & 2.4 & 1362 & 4816 & 7214 & 1513 & 10,388 & 9250 & 179 & 1179 & 1156 & 3.22 & 0.77 & 0.73 & 0.1 & 0.4 & 0.3 \\
\hline NC State University & PI 471938 & IV & 657 & 3505 & 2933 & 104 & 482 & 406 & 0.5 & 0.4 & 0.4 & 1.3 & 5.3 & 4.5 & 2756 & 6232 & 8144 & 1615 & 14,331 & 14,341 & 179 & 1714 & 1512 & 1.50 & 0.64 & 0.62 & 0.1 & 0.4 & 0.3 \\
\hline
\end{tabular}


Table 2. Cont

\begin{tabular}{|c|c|c|c|c|c|c|c|c|c|c|c|c|c|c|c|c|c|c|c|c|c|c|c|c|c|c|c|c|c|}
\hline \multirow{2}{*}{ Company } & \multirow[t]{2}{*}{ Cultivar } & \multirow[t]{2}{*}{ MG } & \multicolumn{3}{|c|}{ CRL, cm plant ${ }^{-1}$} & \multicolumn{3}{|c|}{ RSA, $\mathrm{cm}^{2}$ plant $^{-1}$} & \multicolumn{3}{|c|}{ RAD, cm } & \multicolumn{3}{|c|}{$\mathrm{RV}, \mathrm{cm}^{3}$ plant $^{-1}$} & \multicolumn{3}{|c|}{ RNT, no. plant ${ }^{-1}$} & \multicolumn{3}{|c|}{ RNF, no. plant $^{-1}$} & \multicolumn{3}{|c|}{ RNC, no. plant ${ }^{-1}$} & \multicolumn{3}{|c|}{$\mathrm{R} / \mathrm{S}$} & \multicolumn{3}{|c|}{$\mathrm{RW}, \mathrm{g}$ plant $^{-1}$} \\
\hline & & & LT & OT & HT & LT & OT & HT & LT & OT & HT & LT & OT & HT & LT & OT & HT & LT & от & HT & LT & OT & HT & LT & OT & HT & LT & от & HT \\
\hline University of Missouri & R01-416F & IV & 635 & 3828 & 3110 & 88 & 515 & 422 & 0.4 & 0.4 & 0.4 & 1.0 & 5.5 & 4.6 & 1879 & 5635 & 5029 & 1869 & 16,496 & 14,412 & 199 & 1952 & 1660 & 1.43 & 0.79 & 0.70 & 0.1 & 0.4 & 0.3 \\
\hline & Mean & IV & 621 & 2725 & 2555 & 100 & 379 & 348 & 0.5 & 0.4 & 0.4 & 1.3 & 4.2 & 3.8 & 1825 & 5785 & 5926 & 1357 & 9802 & 10,571 & 158 & 1162 & 1212 & 2.1 & 0.8 & 0.8 & 0.1 & 0.3 & 0.3 \\
\hline Asgrow & AG5332 & $\mathrm{V}$ & 771 & 2660 & 2245 & 125 & 355 & 313 & 0.5 & 0.4 & 0.4 & 1.6 & 3.8 & 3.5 & 2007 & 6025 & 4339 & 1402 & 8549 & $8272 \quad 1$ & 164 & 1052 & 975 & 2.77 & 0.77 & 0.96 & 0.1 & 0.3 & 0.3 \\
\hline Progeny & PR 5333 & $\mathrm{~V}$ & 675 & 3501 & 2683 & 100 & 480 & 345 & 0.5 & 0.4 & 0.4 & 1.2 & 5.2 & 3.5 & 2589 & 5115 & 6602 & 1714 & 14,145 & 11,916 & 199 & 1823 & 1456 & 1.92 & 0.82 & 0.61 & 0.1 & 0.4 & 0.3 \\
\hline USDA-ARS & JTN-5110 & $\mathrm{v}$ & 792 & 3676 & 2936 & 117 & 514 & 405 & 0.5 & 0.4 & 0.4 & 1.4 & 5.8 & 4.5 & 2800 & 6792 & 6115 & 1900 & 17,403 & 13,6162 & 231 & 1853 & 1227 & 1.64 & 0.69 & 0.92 & 0.1 & 0.5 & 0.4 \\
\hline Go Soy Genetics Optimized & LELAND & $\mathrm{V}$ & 681 & 3814 & 3105 & 111 & 522 & 398 & 0.5 & 0.4 & 0.4 & 1.4 & 5.7 & 4.1 & 1747 & 7842 & 9836 & 1192 & 16,821 & 13,978 & 140 & 1816 & 1704 & 2.86 & 0.71 & 0.73 & 0.1 & 0.3 & 0.3 \\
\hline Delta Grow Seeds Com. Inc. & DG5067LL & $\mathrm{v}$ & 802 & 3313 & 2232 & 122 & 436 & 317 & 0.5 & 0.4 & 0.4 & 1.5 & 4.6 & 3.6 & 2005 & 6564 & 7167 & 1892 & 12,744 & $9638 \quad 2$ & 247 & 1635 & 941 & 2.14 & 1.01 & 0.70 & 0.1 & 0.3 & 0.3 \\
\hline Go Soy Genetics Optimized & $5115 \mathrm{LL}$ & $\mathrm{v}$ & 615 & 3417 & 1848 & 117 & 484 & 277 & 0.5 & 0.5 & 0.5 & 1.4 & 5.5 & 3.3 & 1143 & 8448 & 5117 & 1166 & $11,630 \mathrm{c}$ & 6408 & 142 & 1408 & 647 & 2.00 & 1.04 & 0.63 & 0.1 & 0.4 & 0.2 \\
\hline Dyna-Gro Seed & $\begin{array}{l}\text { S55LS75 } \\
\end{array}$ & $\mathrm{V}$ & 589 & 2689 & 3081 & 93 & 373 & 438 & 0.5 & 0.4 & 0.5 & 1.2 & 4.1 & 5.0 & 1303 & 4594 & 6105 & 1206 & 9725 & 12,816 & 174 & 1158 & 1437 & 1.60 & 0.59 & 0.78 & 0.1 & 0.3 & 0.4 \\
\hline Bayer Credenz & CZ 5242 LL & $\mathrm{v}$ & 612 & 2388 & 2676 & 92 & 310 & 367 & 0.5 & 0.4 & 0.4 & 1.1 & 3.2 & 4.0 & 1617 & 6525 & 5050 & 1230 & 8460 & 10,822 & 148 & 923 & 1320 & 1.89 & 0.72 & 0.71 & 0.1 & 0.2 & 0.3 \\
\hline Bayer Credenz & CZ5225 LL & $\mathrm{v}$ & 424 & 2426 & 2552 & 61 & 317 & 351 & 0.5 & 0.4 & 0.4 & 0.7 & 3.3 & 3.9 & 1303 & 6817 & 5478 & 909 & 9166 & $9809 \quad 1$ & 100 & 960 & 1085 & 1.50 & 0.62 & 0.61 & 0.0 & 0.2 & 0.3 \\
\hline Delta Grow Seeds Com. Inc. & DG 5170RR2/STS & $\mathrm{V}$ & 530 & 3219 & 2985 & 87 & 456 & 441 & 0.5 & 0.4 & 0.5 & 1.1 & 5.1 & 5.2 & 1544 & 6606 & 6300 & 877 & 11,589 & 13,906 & 103 & 1313 & 1333 & 1.62 & 0.79 & 0.77 & 0.1 & 0.4 & 0.3 \\
\hline REV Brand Seeds & $51 \mathrm{~A} 56$ & $\mathrm{~V}$ & 677 & 2747 & 2386 & 109 & 368 & 296 & 0.5 & 0.4 & 0.4 & 1.4 & 3.9 & 2.9 & 1405 & 8443 & 10,390 & 1771 & 9838 & $7798 \quad 1$ & 190 & 1230 & 873 & 1.57 & 0.81 & 0.55 & 0.1 & 0.3 & 0.3 \\
\hline DuPont Pioneer & P52T50R & $\mathrm{v}$ & 625 & 2963 & 2928 & 98 & 391 & 372 & 0.5 & 0.4 & 0.4 & 1.2 & 4.1 & 3.8 & 1211 & 6504 & 7084 & 1276 & 11,012 & 12,055 & 158 & 1391 & 1481 & 2.88 & 0.76 & 0.69 & 0.1 & 0.3 & 0.3 \\
\hline Syngenta United States & S55-Q3 & $\mathrm{V}$ & 445 & 2347 & 2562 & 70 & 298 & 328 & 0.5 & 0.4 & 0.4 & 0.9 & 3.0 & 3.4 & 1742 & 7492 & 7220 & 1155 & 8729 & 10,726 & 115 & 1011 & 1247 & 0.86 & 0.80 & 0.81 & 0.1 & 0.2 & 0.3 \\
\hline Syngenta United States & 556-M8 & $\mathrm{V}$ & 761 & 2736 & 2298 & 113 & 349 & 301 & 0.5 & 0.4 & 0.4 & 1.3 & 3.6 & 3.2 & 1534 & 10,121 & 8450 & 1770 & 10,547 & $9242 \quad 2$ & 235 & 1222 & 1049 & 1.10 & 1.05 & 0.90 & 0.0 & 0.2 & 0.2 \\
\hline Go Soy Genetics Optimized & $5214 \mathrm{GTS}$ & $\mathrm{v}$ & 491 & 2581 & 2986 & 76 & 353 & 379 & 0.5 & 0.4 & 0.4 & 0.9 & 3.9 & 3.8 & 1864 & 3850 & 5892 & 877 & 9461 & 12,930 & 116 & 1129 & 1610 & 1.80 & 0.59 & 0.61 & 0.1 & 0.3 & 0.3 \\
\hline Armor & 55-R68 & $\mathrm{V}$ & 659 & 3108 & 2961 & 99 & 399 & 388 & 0.5 & 0.4 & 0.4 & 1.2 & 4.1 & 4.1 & 2160 & 5362 & 4301 & 1193 & 10,938 & 13,484 & 164 & 1486 & 1671 & 1.43 & 0.72 & 0.69 & 0.1 & 0.3 & 0.3 \\
\hline Progeny Ag Products & P 5226RYS & $\mathrm{V}$ & 717 & 3336 & 2889 & 112 & 469 & 395 & 0.5 & 0.4 & 0.4 & 1.4 & 5.3 & 4.3 & 1441 & 4898 & 6982 & 1660 & 11,885 & 11,4452 & 217 & 1505 & 1274 & 3.00 & 0.82 & 0.66 & 0.1 & 0.3 & 0.3 \\
\hline Mycogen Seeds & 5N523R2 & $\mathrm{V}$ & 692 & 2981 & 2039 & 115 & 441 & 293 & 0.5 & 0.5 & 0.5 & 1.5 & 5.2 & 3.4 & 3097 & 3801 & 5335 & 1791 & 9946 ? & 7093 & 164 & 1257 & 780 & 3.43 & 0.88 & 0.87 & 0.1 & 0.3 & 0.3 \\
\hline Dyna-Gro seed & S56RY84 & $\mathrm{V}$ & 864 & 3195 & 3028 & 132 & 457 & 433 & 0.5 & 0.5 & 0.5 & 1.6 & 5.2 & 5.0 & 1292 & 4672 & 6125 & 1973 & 12,079 & 15,0972 & 293 & 1440 & 1553 & 2.33 & 0.65 & 0.77 & 0.1 & 0.3 & 0.4 \\
\hline Croplan WinField United & R2C5225S & $\mathrm{V}$ & 737 & 3008 & 2750 & 120 & 430 & 379 & 0.5 & 0.5 & 0.4 & 1.6 & 4.9 & 4.2 & 1729 & 5019 & 5144 & 1654 & 10434 & 10,673 & 186 & 1266 & 1190 & 2.78 & 0.72 & 0.76 & 0.1 & 0.4 & 0.4 \\
\hline Bayer Credenz & CZ 5375 RY & $\mathrm{V}$ & 594 & 3017 & 3530 & 104 & 428 & 486 & 0.5 & 0.5 & 0.4 & 1.5 & 4.8 & 5.3 & 1782 & 5153 & 7038 & 1214 & 11,698 & 16,497 & 130 & 1312 & 1861 & 1.46 & 0.90 & 0.87 & 0.1 & 0.3 & 0.4 \\
\hline REV Brand Seeds & $57 \mathrm{R} 21$ & $\mathrm{~V}$ & 553 & 2342 & 2450 & 83 & 310 & 333 & 0.5 & 0.4 & 0.4 & 1.0 & 3.3 & 3.6 & 1815 & 10,931 & 6604 & 1303 & 8993 & 9743 & 166 & 907 & 1103 & 1.25 & 0.70 & 0.93 & 0.1 & 0.3 & 0.3 \\
\hline Syngenta United States & S58-Z4 & $\mathrm{v}$ & 395 & 2424 & 2092 & 63 & 323 & 282 & 0.5 & 0.4 & 0.4 & 0.8 & 3.4 & 3.0 & 1345 & 6649 & 4403 & 684 & 7774 & 7873 & 81 & 933 & 934 & 1.63 & 0.77 & 0.79 & 0.0 & 0.2 & 0.3 \\
\hline \multirow[t]{2}{*}{ Dyna-Gro Seed } & S57RY26 & $\mathrm{v}$ & 914 & 3488 & 3736 & 134 & 485 & 493 & 0.5 & 0.4 & 0.4 & 1.6 & 5.4 & 5.2 & 1487 & 4933 & 7876 & 2420 & 15,1772 & 20,0923 & 359 & 1740 & 2150 & 3.00 & 0.76 & 0.73 & 0.1 & 0.4 & 0.3 \\
\hline & Mean & $\mathrm{V}$ & 651 & 2974 & 2707 & 102 & 406 & 367 & 0.5 & 0.4 & 0.4 & 1.3 & 4.4 & 4.0 & 1748 & 6382 & 6456 & 1426 & 11,198 & \begin{tabular}{|l|l|}
11,497 \\
\end{tabular} & 176 & 1324 & 1288 & 2.0 & 0.8 & 0.8 & 0.1 & 0.3 & 0.3 \\
\hline Mean & & & 628 & 2798 & 2590 & 101 & 388 & 353 & 0.5 & 0.4 & 0.4 & 1.3 & 4.3 & 3.8 & 1791 & 5928 & 6037 & 1367 & 10,202 & 10,761 & 162 & 1204 & 1226 & 2.06 & 0.81 & 0.77 & 0.1 & 0.3 & 0.3 \\
\hline \multicolumn{30}{|l|}{ + ANOVA } \\
\hline MG & & & & $* * *$ & & & $* * *$ & & & $* * *$ & & & ns & & & $* * *$ & & & $* * *$ & & & $* * *$ & & & ns & & & ns & \\
\hline CUL & & & $* * *$ & $* * *$ & $* * *$ & $* * *$ & $* * *$ & $* * *$ & * & ** & $* *$ & ns & $* * *$ & $* * *$ & $* * *$ & $* * *$ & $* * *$ & $* * *$ & $* * *$ & $* * *$ & $* * *$ & $* * *$ & $* * *$ & $* * *$ & $* * *$ & $* * *$ & $* *$ & ns & ns \\
\hline TT & & & & $* * *$ & & & $* * *$ & & & $* * *$ & & & $* * *$ & & & $* * *$ & & & $* * *$ & & & $* * *$ & & & $* * *$ & & & & **** \\
\hline LT & & & & $* * *$ & & & $* * *$ & & & $* * *$ & & & $* * *$ & & & $* * *$ & & & $* * *$ & & & $* * *$ & & & $* * *$ & & & & $* * *$ \\
\hline $\mathrm{HT}$ & & & & $* * *$ & & & $* * *$ & & & $* * *$ & & & $* * * *$ & & & $* * *$ & & & $* * *$ & & & $* * *$ & & & $* * *$ & & & & ns \\
\hline MG $\times$ TT & & & & ns & & & ns & & & $\mathrm{ns}$ & & & ns & & & $* * *$ & & & $* * *$ & & & $* *$ & & & ns & & & & ns \\
\hline CUL $\times$ TT & & & & $* * *$ & & & $* * *$ & & & ns & & & $* * *$ & & & $* * *$ & & & $* * *$ & & & $* * * *$ & & & * & & & & ns \\
\hline
\end{tabular}

$\dagger^{*}, * *, * * *$, and ns representing significance at the $p \leq 0.05, p \leq 0.01, p \leq 0.001$, and non-significant $(p \geq 0.05)$, respectively. CUL, cultivar; MG, maturity group; TT, temperature treatment; $\mathrm{LT}$, low-temperature treatment; $\mathrm{HT}$, high-temperature treatment; $\mathrm{OT}$, optimum temperature treatment; $\mathrm{R} / \mathrm{S}$, root to shoot ratio. 


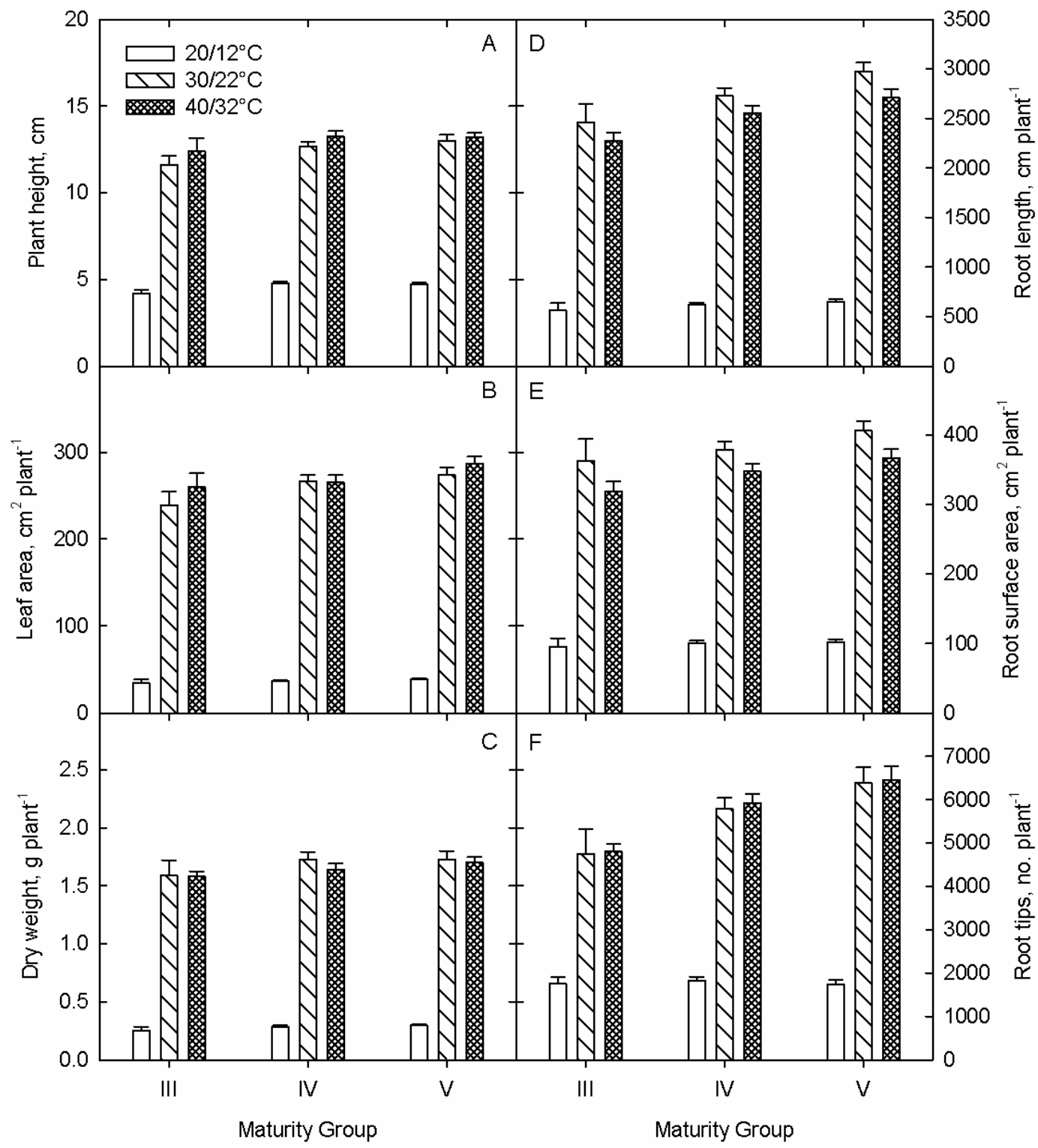

Figure 1. Temperature and maturity group interaction for (A) plant height, (B) leaf area, (C) dry weight, (D) root length, (E) root surface area, and (F) root tips for soybean 64 cultivars harvested 20 days after sowing. Data shows mean $+\mathrm{SE}$.

\subsubsection{Root Parameters}

The effects of TTs were significant in all the measured root parameters (Table 2). Further, cultivars varied significantly across TTs for all the measured root parameters, except root volume (RV) at LT, and root weights $(\mathrm{RW})$. Cultivar $\mathrm{x}$ TT interaction was also significant $(p<0.001)$ for all the root parameters, except root average diameter (RAD) and RW (Table 2). Maturity groups significantly differed for root traits, except for RV, RW, and R/S, while interaction effect of MG with TTs was significant only for lateral root development (i.e., RNF, RNC, and RNT) (Table 2).

\section{Root Growth}

Unlike shoot growth, root growth parameters, including CRL, RSA, and RV showed decline under stress conditions than control (Table 2). The mean CRL was obtained highest at OT $(2798.06 \mathrm{~cm})$, followed by HT $(2590.36 \mathrm{~cm})$, and LT (627.65), respectively (Table 2). The percent decline in mean CRL was higher at LT $(77.5 \%)$ than HT $(7.4 \%)$. Among 64 soybean cultivars, S39-T3 showed significantly 
highest $(84.09 \%)$, and 5N393R2 showed significantly lowest $(64.6 \%)$ decline in CRL at LT when compared to OT (Table 2). Overall, the mean CRL was significantly higher for MG V $(2110.72 \mathrm{~cm})$ followed by MG IV $(1973.84 \mathrm{~cm})$ and MG III $(1766.79 \mathrm{~cm})$. On an average across cultivars, the percent decline in RSA at LT and HT were $74 \%$ and $9 \%$, respectively. The highest and lowest value for RSA were obtained in LELAND at OT, and CZ 5225 LL at LT, respectively (Table 2). Unlike LT effects, some cultivars including 5N393R2, IREANE, and ELLIS had greater values for CRL and RSA under HT when compared to OT. The cultivars belonging to MG V had significantly higher RSA on an average (Figure 1E), with no differences observed between MG III and IV $(p>0.05)$. The percent increases of RSA were 6,11 , and $13 \%$ for the cultivars from MG V compared to MG III at low, optimum, and high temperature conditions (Figure 1E). Interestingly, the mean value of RAD was significantly greater at LT $(0.51 \mathrm{~mm})$ than OT $(0.44 \mathrm{~mm})$ or HT $(0.43 \mathrm{~mm})$ across the tested cultivars (Table 2). Contrasting to CRL, MG III showed significantly higher mean RSA $(0.49 \mathrm{~mm})$ followed by MG $4(0.46 \mathrm{~mm})$ and MG5 $(0.45 \mathrm{~mm})$, respectively (Table 2$)$.

\section{Root Development}

In contrast to root growth, the mean values of root development parameters, including RNT, RNF, and RNC, significantly increased with increasing temperatures from low to high (Table 2). On an averaged across cultivars, the values for RNF, RNT, and RNC ranged from 1791 to 6037, 1367 to 10,761, and 165 to 1226, respectively, across TTs (Table 2). Cultivars 51A56 and 5115LL showed maximum and minimum values for RNT, while cultivars S57RY26 and S39-T3 showed maximum and minimum values for RNF as well as RNC, respectively, across TTs (Table 2). Likewise observed for shoot parameters, the study observed a reduction in root development (i.e., RNT, RNF, and RNC) with an increase from optimum to high temperatures in some cultivars, including GS45R216, S45-W9, P47T36R, and AR4705 (Table 2). Also, the strong effect of MG was observed in lateral root development, such that MG V cultivars showed greater lateral root numbers than MG III and IV (Figure 1F). However, the effects were modified with increasing temperatures.

\subsubsection{Plant-Component Dry Weights}

The study observed significant effects of LT and HT on leaf dry weight (LW), while stem dry weights (SW) and root dry weights (RW) were only affected under LT (Tables 1 and 2). No effects of MG or MG $\times$ TT were found on dry weights (Tables 1 and 2). The decline in LW was higher under LT effects $(82.5 \%)$ than under HT effects $(5.7 \%)$ compared to OT when averaged across cultivars. However, cultivars varied significantly for LW under LT and OT, but not under HT (Table 1). The LW ranged from $0.05 \mathrm{~g}$ in GS47R216 at LT to $2.67 \mathrm{~g}$ in P $4757 \mathrm{RY}$ at OT. The mean SW and RW showed a reduction of $90.6 \%$ and $77 \%$ under LT stress, respectively when compared to OT. Among tested cultivars, the maximum reduction was observed in R2C4775 (94.2\%) and S39-T3 (86.7\%) for SW and RW, respectively under LT effects (Tables 1 and 2). Total dry weight (TW) calculated by summation of LW, SW, and RW was significantly reduced under LT effects by $83.4 \%$. Further, cultivar $\times$ TT interaction was significant for TW such that TW varied between $0.14 \mathrm{~g}$ in S45-W9 at LT and $3.43 \mathrm{~g}$ in P $4757 \mathrm{RY}$ at OT (Table 1).

\subsubsection{Physiological Parameters}

The study observed significant cultivar, MG, and TT effects on chlorophyll measured and expressed as SPAD values $(p<0.001)$. The SPAD values significantly increased from LT to HT, ranging from 26.86 to 41.93 , on an average across the cultivars (Table 1). Among tested cultivars, the maximum and minimum SPAD values were observed in S48RS53 (38.7) and 55-R68 (31.7), respectively across TTs. Overall, MG III showed significantly higher SPAD values than MG IV and V. However the interaction effects were not significant for SPAD value $(p>0.05)$. Similarly, mean canopy temperatures were significantly $(p<0.001)$ highest under HT $\left(34.9^{\circ} \mathrm{C}\right)$, followed by OT $\left(29.8^{\circ} \mathrm{C}\right)$, 
and LT $\left(24.9^{\circ} \mathrm{C}\right)$, respectively, across cultivars, with no difference $(p>0.05)$ observed among cultivars and maturity groups.

\subsection{Cumulative Stress Response Indices}

Cumulative stress response indices for high temperature (CHTRI) varied from 13.02 (heat sensitive) to 26.28 (heat tolerant) across 64 soybean cultivars. Based on CHTRI values and an increment of $1.0 \mathrm{SD}$, nine cultivars were identified as highly sensitive, 30 were moderately sensitive, 17 were moderately tolerant, and eight were highly tolerant to HT, among the tested cultivars (Table 3). Cultivar 45A-46 showed the highest tolerance, and 5115LL showed the highest sensitivity to HT effects, respectively. Further, CHTRI showed a positive and significant correlation $(p>0.001)$ to CHTRI calculated for root parameters $\left(r^{2}=0.91\right)$ and shoot parameters $\left(r^{2}=0.70\right)$ separately (Figure 2$)$.

Table 3. Classification of soybean cultivars into high-temperature tolerance groups based on cumulative high-temperature response index (CHTRI; unitless), along with individual scores in parenthesis.

\begin{tabular}{|c|c|c|c|}
\hline $\begin{array}{c}\text { Heat Sensitive } \\
\text { (CHTRI }=13.20 \text { to } 15.32)\end{array}$ & $\begin{array}{l}\text { Moderately Heat-Sensitive } \\
\text { (CHTRI = 15.33 to 17.62) }\end{array}$ & $\begin{array}{l}\text { Moderately Heat-Tolerant } \\
\text { (CHTRI = 17.63 to 19.91) }\end{array}$ & $\begin{array}{l}\text { Heat Tolerant } \\
\text { (CHTRI > 19.92) }\end{array}$ \\
\hline $\begin{array}{c}\text { 5115LL (13.02) } \\
\text { S47-K5 (14.00) } \\
\text { S45-W9 }(14.77) \\
483 C(14.91) \\
\text { 38 R10 (14.92) } \\
\text { R01-416F (15.06) } \\
\text { JTN-5110 (15.16) } \\
\text { S48RS53 (15.24) } \\
\text { DG 4825RR2/STS (15.25) }\end{array}$ & $\begin{array}{c}\text { GS45R216 (15.47) } \\
\text { 5N424R2 (15.05) } \\
\text { GT-476CR2 (15.58) } \\
\text { AG4632 (15.66) } \\
\text { 5N523R2 (15.66) } \\
\text { P47T36R (15.94) } \\
\text { P 4588RY (15.97) } \\
\text { P41T33R (16.03) } \\
\text { P 5333 RY (16.09) } \\
\text { LELAND (16.25) } \\
\text { GS47R216 (16.38) } \\
\text { DG 4680RR2 (16.41) } \\
\text { 31RY45 (16.46) } \\
\text { PI 471938 (16.50) } \\
\text { P 5226 RYS (16.57) } \\
\text { R2C5225S (16.61) } \\
\text { 51A56 (16.71) } \\
\text { P 4757 RY (16.38) } \\
\text { AR4705 (16.90) } \\
\text { AG5332 (16.93) } \\
\text { 55-R68 (17.06) } \\
\text { S39-C4 (17.10) } \\
\text { DG 5170 RR2/STS (17.27) } \\
\text { P52T50R (17.38) } \\
\text { 57R21 (17.44) } \\
\text { 4814GTS (17.51) } \\
\text { DG 5067 LL (17.52) } \\
\text { S56-M8 (17.54) } \\
\text { 32y39 (17.54) } \\
\text { 4714LL (17.59) }\end{array}$ & $\begin{array}{c}\text { DG4781LL (17.75) } \\
\text { S39-T3 (18.01) } \\
\text { S56RY84 (18.03) } \\
\text { CZ 5225 LL (18.03) } \\
\text { S58-Z4 (18.04) } \\
\text { DB 4911 (18.07) } \\
\text { R2C4775 (18.10) } \\
\text { P 4247 LL (18.69) } \\
\text { S57RY26 (18.79) } \\
\text { CZ 4044 LL (18.83) } \\
\text { 5214GTS (18.91) } \\
\text { 48A26 (19.09) } \\
\text { S55-Q3 (19.25) } \\
\text { 5N490R2 (19.35) } \\
\text { S49LL34 (19.54) } \\
\text { 48L63 (19.73) } \\
\text { S55LS75 (19.81) }\end{array}$ & $\begin{array}{c}\text { IREANE (20.25) } \\
\text { CZ 4898 RY (20.59) } \\
\text { CZ 5242 LL (20.84) } \\
\text { CZ 5375 RY (20.88) } \\
\text { ELLIS (21.16) } \\
\text { 5N393R2 (21.21) } \\
\text { CZ 4181 RY (24.17) } \\
\text { 45A46 (26.28) }\end{array}$ \\
\hline
\end{tabular}

Cumulative stress response indices for low temperatures classified 21 cultivars as cold sensitive, 32 cultivars as moderately cold sensitive, six cultivars as moderately cold tolerant, and five cultivars as cold tolerant, based on the means and SD. Cultivars CZ 5225 LL and GS47R216 were identified as most sensitive and tolerant to LT, respectively (Table 4). Unlike CHTRI, CLTRI has had a poor correlation to CLTRI calculated for the shoot $\left(r^{2}=0.05\right)$ and root parameters $\left(r^{2}=0.38\right)$ separately (Figure 3$)$. However, CLTRI had a significant and positive correlation with CHTRI $\left(p<0.001 ; r^{2}=0.96\right)$ (Figure 4$)$. 


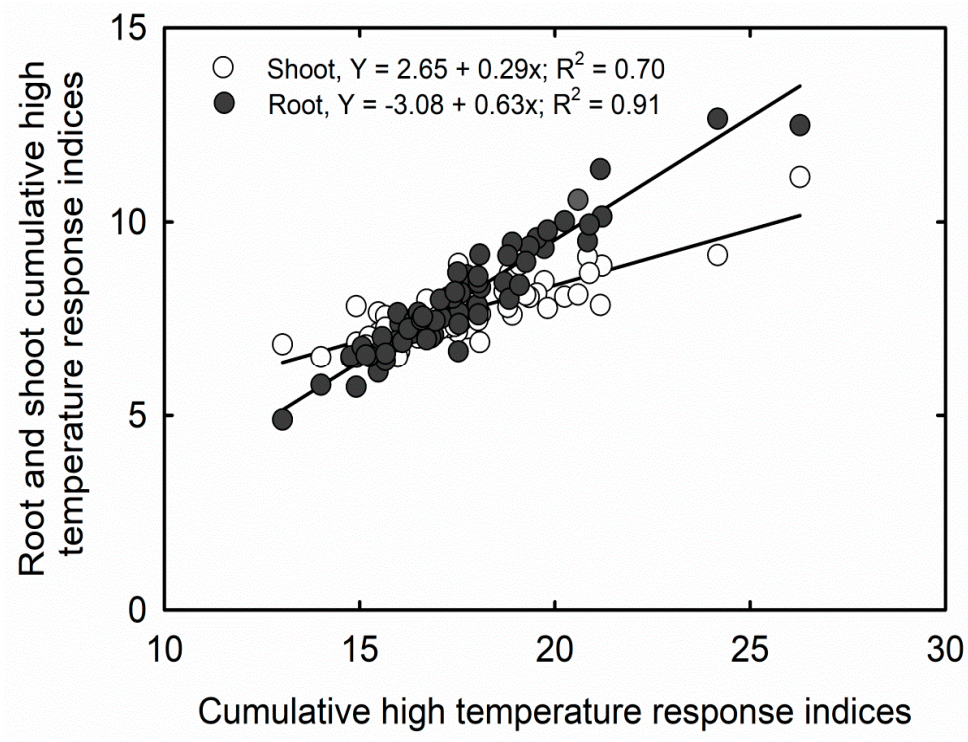

Figure 2. Correlation between cumulative high-temperature response indices and cumulative high-temperature response indices calculated for root and shoot parameters separately among 64 soybean cultivars, measured at 20 days after sowing.

Table 4. Classification of soybean cultivars into cold tolerance groups based on total low-temperature response index (CLTRI; unitless), along with individual scores in parenthesis.

\begin{tabular}{|c|c|c|c|}
\hline Cold-Sensitive & $\begin{array}{l}\text { Moderate Cold } \\
\text { Sensitive }\end{array}$ & Moderate Cold Tolerant & Cold Tolerant \\
\hline$($ CLTRI = 6.12 to 6.94$)$ & $($ CLTRI $=6.95$ to 7.76$)$ & (CLTRI = 7.77 to 8.58$)$ & (CLTRI > 8.59) \\
\hline CZ 5225 LL (6.12) & S47-K5 (6.95) & 5N523R2 (7.94) & 4714LL (8.68) \\
\hline CZ 4044 LL (6.16) & DG 4680RR2 (6.98) & P52T50R (7.99) & 5N393R2 (8.94) \\
\hline R01-416F (6.21) & R2C4775 (6.99) & S48RS53 (8.10) & AG5332 (9.42) \\
\hline 57R21 (6.29) & CZ 5375 RY (7.00) & 45A46 (8.15) & GT-476CR2 (9.83) \\
\hline PI 471938 (6.32) & P 5226 RYS (7.00) & DG4781LL (8.29) & GS47R216 (10.34) \\
\hline S39-T3 (6.39) & S58-Z4 (7.00) & P47T36R (8.56) & \\
\hline 48A26 (6.54) & R2C5225S (7.01) & & \\
\hline AG4632 (6.63) & 38 R10 (7.02) & & \\
\hline CZ 4898 RY (6.63) & S55-Q3 (7.03) & & \\
\hline 5115LL (6.66) & $483 C(7.12)$ & & \\
\hline DG 5170 RR2/STS (6.67) & CZ 4181 RY (7.13) & & \\
\hline S39-C4 (6.75) & S56-M8 (7.15) & & \\
\hline P 4588RY (6.76) & 5214GTS (7.16) & & \\
\hline P 5333 RY (6.77) & S45-W9 (7.20) & & \\
\hline LELAND (6.78) & DB 4911 (7.21) & & \\
\hline S55LS75 (6.81) & 32y39 (7.25) & & \\
\hline 5N424R2 (6.83) & GS45R216 (7.25) & & \\
\hline JTN-5110 (6.86) & 51A56 (7.28) & & \\
\hline 4814GTS (6.86) & AR4705 (7.29) & & \\
\hline 31RY45 (6.90) & IREANE (7.30) & & \\
\hline \multirow[t]{12}{*}{ 55-R68 (6.91) } & P 4247 LL (7.37) & & \\
\hline & DG 4825RR2/STS (7.38) & & \\
\hline & $48 \mathrm{~L} 63(7.40)$ & & \\
\hline & S57RY26 (7.40) & & \\
\hline & P 4757 RY (7.42) & & \\
\hline & CZ 5242 LL (7.51) & & \\
\hline & DG 5067 LL (7.53) & & \\
\hline & ELLIS (7.61) & & \\
\hline & P41T33R (7.61) & & \\
\hline & 5N490R2 (7.65) & & \\
\hline & S56RY84 (7.66) & & \\
\hline & S49LL34 (7.71) & & \\
\hline
\end{tabular}




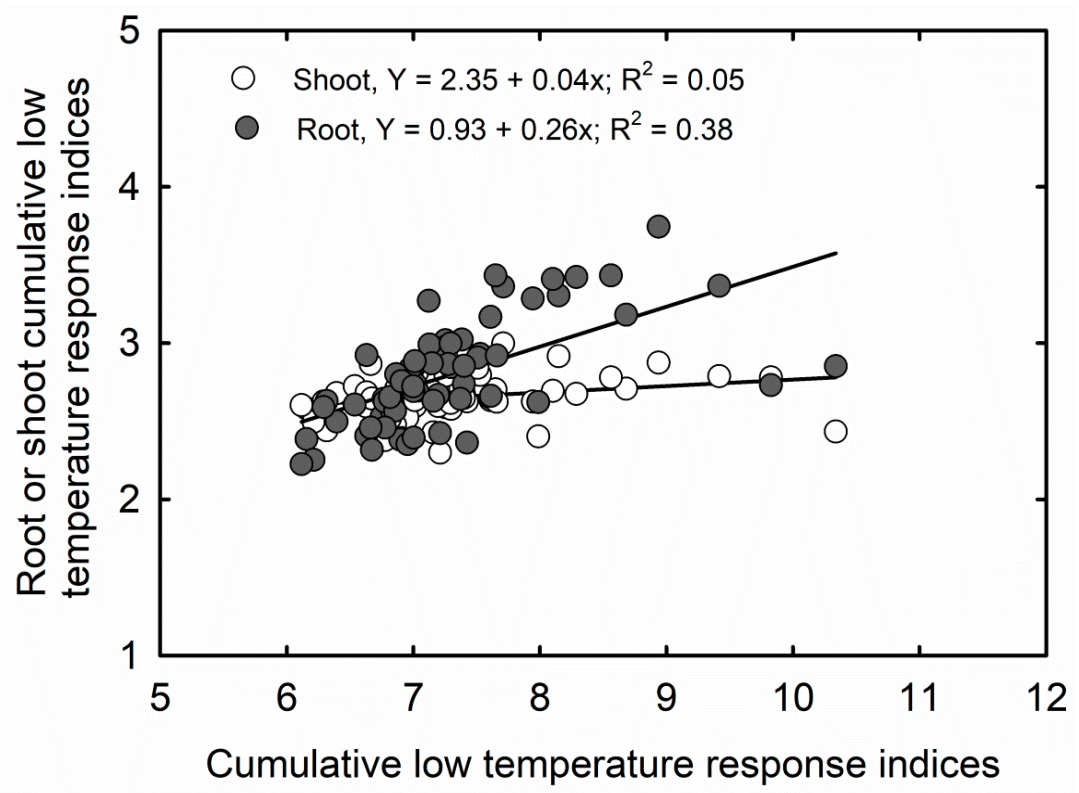

Figure 3. Correlation between cumulative low-temperature response indices and cumulative low-temperature response indices calculated for root and shoot parameters separately among 64 soybean cultivars, measured at 20 days after sowing.

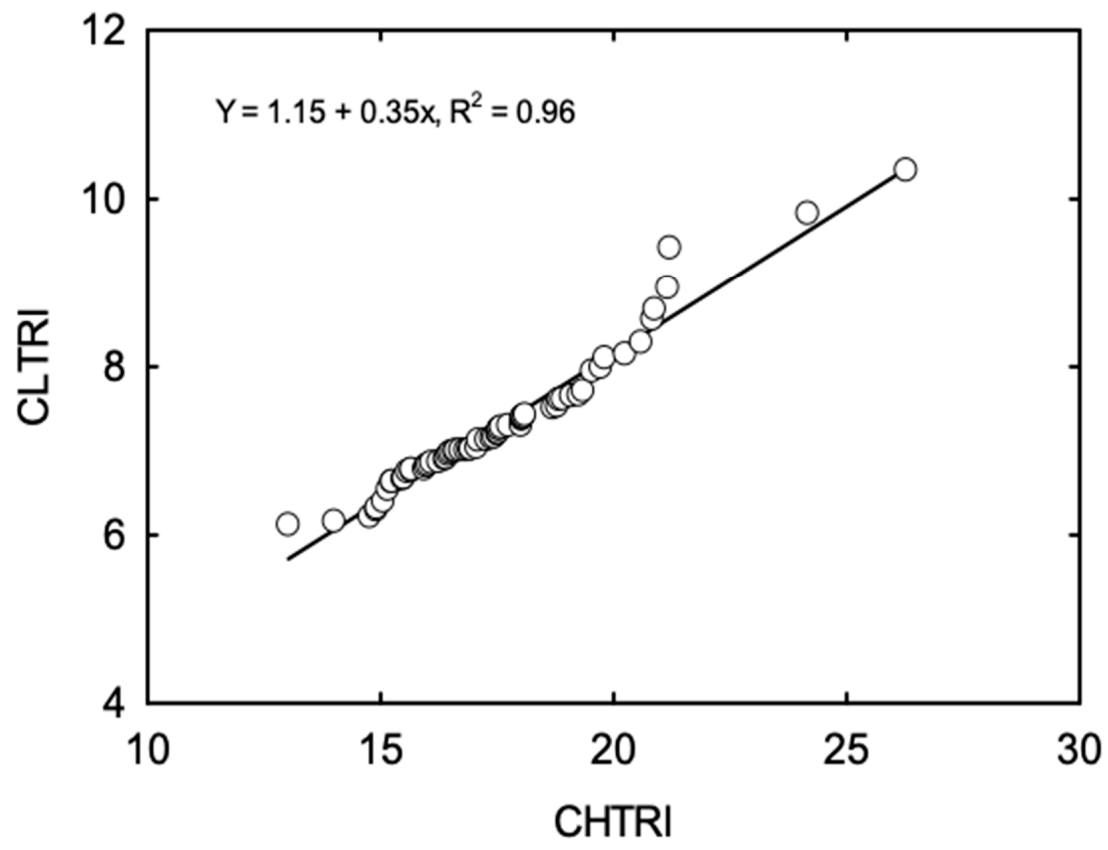

Figure 4. Correlation between cumulative high-temperature response indices (CHTRI) and cumulative low-temperature response indices (CLTRI) among 64 soybean cultivars, measured at 20 days after sowing.

\subsection{Principal Component Analysis (PCA)}

According to the PCA analysis, the first two principal components (PCs) accounted for $56 \%$ of the total variance at low temperature (Figure 5A) while $60 \%$ of the variability was expressed under high temperature (Figure 5B). PC1 accounted for 44 and $49 \%$ of the variance among the cultivars for LT and HT with higher positive loadings for LW, RV, RW, and TW at LT and RL, RSA, RC, and TW at HT (Figure 5). PC2 accounted for an additional 12 and $11 \%$ of the variation with the AD, SPAD, and CT at low temperature and AD, CT, and RV at high temperature. Proceeding from both positive and negative 
loadings of PC1 and PC2, 64 soybean cultivars were classified into three main groups as tolerant, moderate, and sensitive. Within this classification, GT476CR2 and 4714LL represented tolerance, 483C, S56RY84, S57RY26, 45A46, and DG4781LL as moderate, and S47K5 and CZ5225LL as sensitive for low temperature (Figure 5). On the other hand, CZ5242LL and 45A46 came under high-temperature tolerant group followed by S56M8, AG5332, PI471938, DB4911, CZ5225LL, and S39T3 as intermediate and 38R10 and S48RS53 as high temperature sensitive.
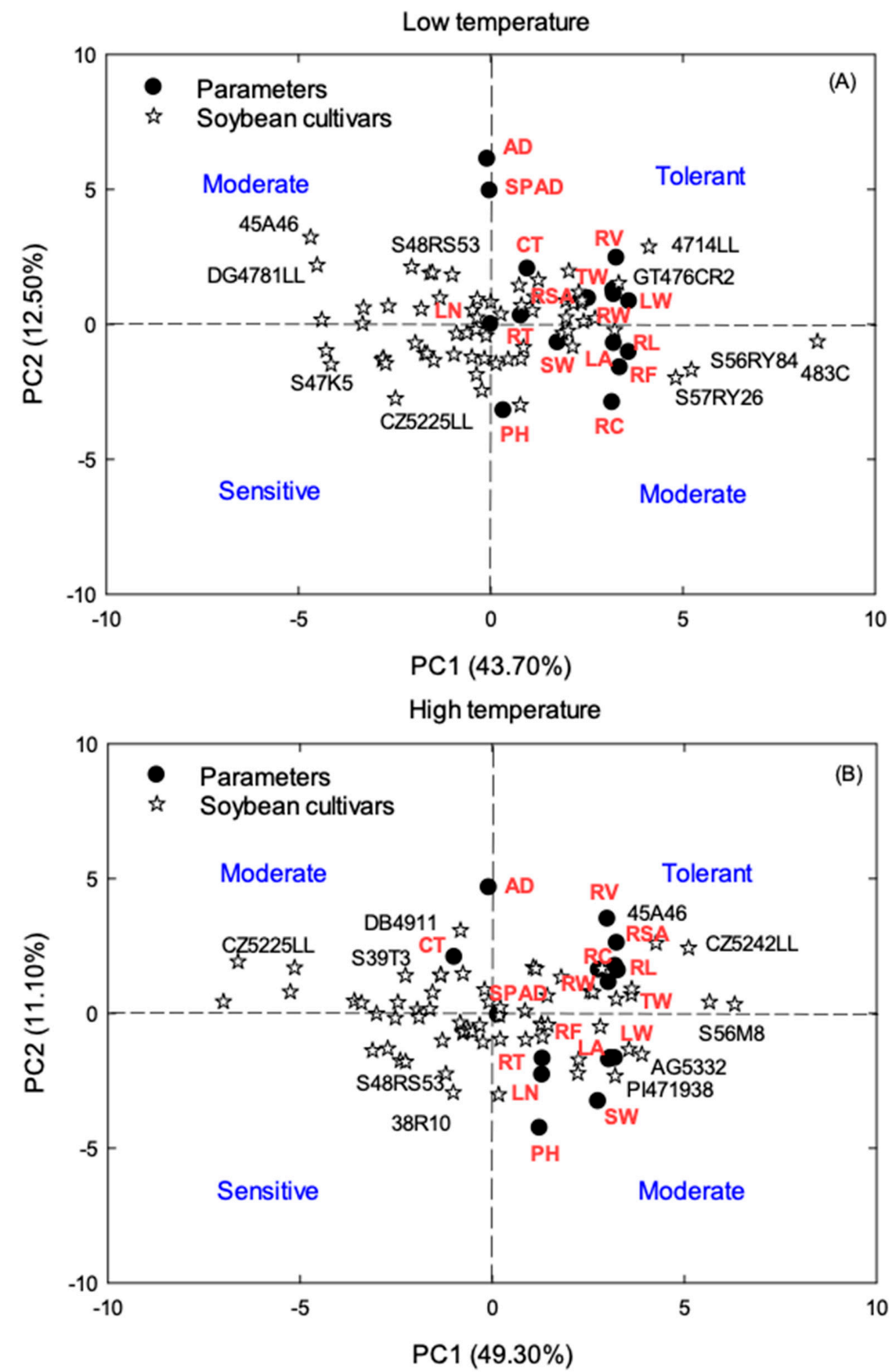

Figure 5. Principal component analysis (PCA) biplot for the first two principal component (PC) scores, PC1, and PC2, related to the classification of 64 soybean cultivars (open stars) for low (A) and high-temperature (B) tolerance. The eigenvectors for the crop traits (solid circles) are superimposed with the PC biplot scores at the similar scale that reflect the contribution of each parameter in the determination of tolerance or the susceptibility towards heat or cold. The eigenvector values were multiplied by ten to obtain a clear and superimposed figure. 


\section{Discussion}

The identification of LT and HT tolerance in soybeans is vital for effective management and production under ESPS and CSPS in US Midsouth. Further, information on cultivar-specific tolerance to a degree of temperatures can be exploited in breeding programs to develop tolerant genotypes that are highly suited for cold or hot environments. Most of the studies in the past have utilized planting date as a criterion to evaluate cultivar's ability to grow under a given production system [2,18-21]. However, several confounding weather factors co-vary during the growing season that limits the results of such studies to validate cultivar's tolerance to low or high-temperature tolerance [16]. The present study is distinct in that it utilizes controlled conditions to identify cultivar-specific tolerance to LT or HT during early-growth, keeping other environments constant. Secondly, this study characterized both shoots as well as root growth and development to determine the temperature tolerance in the soybean cultivars. The present study evaluated soybean cultivars belonging to MG III, IV, and V, which are recommended ideal for the US Midsouth environments based on previous literature describing the interactive effects of agronomic practices, environments, and MG [2,16,19,20,34-36]. The present study showed vigorous seedling growth in cultivars belonging MG IV and V when compared to MG III, which supports recent studies that favored MG IV and V to utilize under ESPS in the US Midsouth [2,19].

Among TTs, LT caused more severe reductions in the shoot, root, and physiological parameters of soybean seedlings than HT. This was expected because, in general, soybean is regarded a warm season crop [9], and considered sensitive to chilling that may occur within a certain range of temperatures during most of the stages of life cycle $[7,16,25]$. The highest damage from chilling injury was observed during germination and seedling emergence of soybean, which showed the severity of damage increase linearly with decreasing temperatures, and finally leading to the death of seedlings [6-8]. Also, chilling injury during seedling growth of soybean has been identified as a major constraint in the success of ESPS in the US Midsouth [5]. Cool and wet conditions developed from early season rainfall may hinder germination of April-planted soybeans under ESPS [4,5]. According to Wuebker et al. [26], seeds flooded for one day after the start of imbibition showed $18 \%$ decrease in germination at $15{ }^{\circ} \mathrm{C}$ than at $25{ }^{\circ} \mathrm{C}$. Similar to the present study, the findings on early-season planting (April-May) of other crops grown in US Midsouth such as cotton and corn reported LT as most damaging for seedling growth among various abiotic stress factors $[27,28,30,31]$. The lesser degree of damage from imposed levels of HT further suggests that like most species, soybean also have a higher temperature optimum for vegetative development than reproductive development [11]. Higher mean values for chlorophyll content as well as canopy temperatures under HT effects than LT further strengths the arguments mentioned above. SPAD values and canopy temperatures are important parameters to evaluate plant photosynthetic efficiency and acclimation $[37,38]$. The higher chlorophyll content attributed to higher photosynthetic rate might have positively contributed to greater plant component dry weights observed under HT treatments in this study.

Interestingly, the present study found varied response of shoot and root parameters to the effects of TTs. The shoot growth was more adversely affected under LT but showed rapid increases under HT effects, when compared to OT. This supports previous reports of rapid germination and emergence on late-season planting (May or later) of soybean under CSPS [2-4]. Little is known on the effects on abiotic on the root system of soybeans compared to other major crops such as corn, rice, and cotton of US Midsouth during seedling growth $[27,28,30]$. Root hydraulic conductivity is considered most sensitive to low temperatures irrespective of soil moisture status [39]. The low temperatures can induce assimilate partitioning regarding higher RAD, and lower CRL and RSA to maintain root hydraulic conductivity in plants $[3,27,28]$. Higher mean RAD observed under LT effects in this study was in agreement with Singh et al. [27] and Wijewardana et al. [28] that found significantly greater mean RAD in cotton and corn seedlings under LT effects, respectively. Moreover, in agreeing with previous findings, RAD was negatively correlated with all the other shoot and root parameters (Table S1). Similar to RAD, RS also exhibited a negative correlation (Table S1), however, all the correlations were 
significantly different $(p<0.001)$. Further, increased lateral root numbers (RNF, RNC, and RNT) under HT corroborate the findings of Khaled et al. [3] that showed mean lateral root numbers in soybeans were significantly increased (12.7\%) in CSPS (June planting) compared to ESPS (April planting). Further, increased lateral root number may have positively contributed to increased root biomass (canopy temperatures) observed under HT effects in this study.

According to PCA, RL, RV, TW, and LA were identified as the traits that best described the temperature tolerance in soybean. Similar to the CLTRI and CHTRI procedure, PCA also identified 4714LL and GT476CR2 as cold tolerant, S47K5 and CZ5225LL as cold sensitive, 45A46 and CZ5242LL as heat-tolerant, and S48RS53 as heat sensitive. Therefore, the findings from PCA were in reasonable agreement with the CLTRI and CHTRI methods where all traits were used in the analysis and the classification of soybean cultivars for low- and high-temperature tolerance. Both positive and negative response in the shoot and root parameters under HT effects supports a positive correlation obtained between CLTRI and CHTRI calculated for a shoot or root parameters separately. The cultivars are showing a reduction in a shoot or root parameters under high temperatures ascribed to their low tolerance to imposed levels of HT or vice versa. A strong and positive correlation between CHTRI and CLTRI indicates that temperature treatments operate likewise on seedling growth and development. For instance, cultivars like 5115LL and JTN-5110 were found sensitive to both LT and HT, while cultivars like 5N393R2 and 45A46 showed tolerance to both HT and LT. The identified tolerance among the tested cultivars based on CHTRI and CLTRI will help farmers in selecting cultivars suited best for a specific region as well as a production system followed, with an aim to maximize benefits regarding temperature tolerance.

\section{Conclusions}

Soybean cultivars varied markedly in their response to high and low temperatures during seedling growth stage, however, modified by maturity group. The reduction in growth and development were more pronounced under LT than under at HT during the seedling growth stage of all soybean cultivars. Significant variability existed in the tested cultivars, from same or different MGs, in responses to imposed TTs for the measured parameters. The changes in morphological and physiological growth characteristics can be ascribed to cultivar's degree of tolerance to the imposed level of temperature stresses. Also, the differential response in the shoot and root parameters to TT and MG signifies the importance of understanding both shoot and root system under stress conditions as well production system followed.

Further, cumulative stress response indices and principal component analysis developed to score the cultivars for high or low-temperature tolerance could be exploited in breeding programs to develop genotypes for temperature tolerance. The LT and HT scores of the cultivars along with region-specific yield data would be helpful for producers' to select cultivars best suited for their production system. Additional research in the field is warranted to investigate the relevance of this study and possibly predicting cultivars tolerance to low and high-temperature conditions under ESPS and CSPS, respectively. Also, testing the cultivars for reproductive tolerance to high temperatures will also be needed to identify tolerance to yield-related parameters.

Supplementary Materials: The following are available online at http:/ / www.mdpi.com/2073-4395/9/1/13/s1, Table S1: Pearson correlation matrix showing the relationship among plant height (PH), leaf area (LA), leaf weight (LW), stem weight (SW), root weight (RW), total weight (TW), canopy temperature (CT), chlorophyll content (SPAD), Cumulative root length (RCL), root surface area (RSA), root diameter (RAD), root volume (RV), number of root tips (RT), number of forks (RF), number of crossings (RC), and root/shoot ratio (RS) of 64 soybean cultivars.

Author Contributions: Conceptualization, K.R.R.; Methodology, K.R.R., F.A.A. and C.W.; Software and Formal Analysis, F.A.A., K.R.R. and C.W.; Investigation, K.R.R. and F.A.A.; Resources, K.R.R.; Data Curation, K.R.R.; Writing-Original Draft Preparation B.S. and F.A.A.; Writing-Review \& Editing, F.A.A., B.S., C.W., J.T.I., W.G. and K.R.R.; Supervision, K.R.R.; Project Administration, K.R.R.; Funding Acquisition, K.R.R., J.T.I. and W.G.

Funding: This research was funded by Mississippi Soybean Promotion Board and National Institute Food and Agriculture, NIFA (2016-34263-25763 and MIS 043040) and Mississippi Soybean Promotion Board and MAFES-SRI. 
Acknowledgments: We thank David Brand for technical assistance and graduate students of the Environmental Plant Physiology Lab at Mississippi State University for their support during data collection. This article is a contribution from the Department of Plant and Soil Sciences, Mississippi State University, Mississippi Agricultural, and Forestry Experiment Station.

Conflicts of Interest: The authors declare no conflict of interest

\section{Abbreviations}

\begin{tabular}{|c|c|}
\hline CSPS & conventional soybean production system \\
\hline CLTRI & cumulative stress response indices for low temperature \\
\hline CHTRI & cumulative stress response indices for high temperature \\
\hline $\mathrm{CT}$ & canopy temperatures \\
\hline CRL & cumulative root length \\
\hline DAS & days after sowing \\
\hline ESPS & early soybean production system \\
\hline HT & high temperature \\
\hline ILTRI & individual stress response index for low temperature \\
\hline IHTRI & individual stress response index for high temperature \\
\hline LA & leaf area \\
\hline LW & leaf weight \\
\hline NN & mainstem node number \\
\hline LT & low temperature \\
\hline MG & maturity group \\
\hline $\mathrm{PH}$ & plant height \\
\hline RAD & root average diameter \\
\hline $\mathrm{RNC}$ & number of root crossings \\
\hline $\mathrm{RF}$ & number of root forks \\
\hline RSA & root surface area \\
\hline CT & canopy temperature \\
\hline RNT & number of root tips \\
\hline $\mathrm{R} / \mathrm{S}$ & root and shoot ratio \\
\hline RV & root volume \\
\hline RW & root weight \\
\hline SW & stem weight \\
\hline RNT & number of root tips \\
\hline TW & total weights \\
\hline $\mathrm{TT}$ & temperature treatments \\
\hline
\end{tabular}

\section{References}

1. Hoeft, R.G.; Aldrich, S.R.; Nafziger, E.D.; Johnson, R.R. Modern Corn and Soybean Production, 1st ed.; MCSP Publications: Champaign, IL, USA, 2000.

2. Heatherly, L.G. Midsouthern USA soybean yield affected by maturity group and planting date. Crop Manag. 2005, 4. [CrossRef]

3. Khaled, O.; Washington, E.; Lage, P.; Kantartzi, S.K.; Lightfoot, D.A.; Kassem, M.A. Comparison of early and conventional soybean production systems for yield and other agronomic traits. Atlas J. Plant Biol. 2011, $1,1-5$.

4. $\quad$ Ashlock, L.O.; Klerk, R.; Huitink, G.; Keisling, T.; Vories, E.D. Planting Practices. Arkansas Soybean Handbook MPV 197; University Arkansas Cooperative Extension Service: Little Rock, AR, USA, 2000; pp. 35-49.

5. Heatherly, L.G.; Hodges, H.F. Early soybean production system (ESPS). In Soybean Production in the Midsouth; Heatherly, L.G., Hodges, H., Eds.; CRC Press: Boca Raton, FL, USA, 1999; pp. 103-118.

6. Vertucci, C.W.; Leopold, A.C. Dynamics of imbibition by soybean embryos. Plant Physiol. 1983, 72, $190-193$. [CrossRef] [PubMed] 
7. Duke, S.H.; Schrader, L.E.; Miller, M.G. Low temperature effects on soybean (Glycine max L. Merr. cv. Wells) mitochondrial respiration and several dehydrogenases during imbibition and germination. Plant Physiol. 1977, 60, 716-722. [CrossRef] [PubMed]

8. Hatfield, J.L.; Egli, D.B. Effect of temperature on the rate of soybean hypocotyl elongation and field emergence. Crop Sci. 1974, 14, 423-426. [CrossRef]

9. Skrudlik, G.; Kościelniak, J. Effects of low temperature treatment at seedling stage on soybean growth, development and final yield. J. Agron. Crop Sci. 1996, 176, 111-117. [CrossRef]

10. Tacarindua, C.R.P.; Shiraiwa, T.; Homma, K.; Kumagai, E.; Sameshima, R. The effects of increased temperature on crop growth and yield of soybean grown in a temperature gradient chamber. Field Crops Res. 2013, 154, 74-81. [CrossRef]

11. Hatfield, J.L.; Prueger, J.H. Temperature extremes: Effect on plant growth and development. Weather Clim. Extrem. 2015, 10, 4-10. [CrossRef]

12. Egli, D.B.; Cornelius, P.L. A regional analysis of the response of soybean yield to planting date. Agron. J. 2009, 101, 330-335. [CrossRef]

13. Thuzar, M.; Puteh, A.B.; Abdullah, N.A.P.; Lassim, M.B.M.; Jusoff, K. The effects of temperature stress on the quality and yield of soya bean (Glycine max L.) Merrill. J. Agric. Sci. 2010, 2, 172-179.

14. Heatherly, L.G.; Blaine, A.; Hodges, H.F.; Wesley, R.A.; Buehring, N.; Heatherly, L.G. Variety selection, planting date, row spacing, and seeding rate. In Soybean Production in the Midsouth; Heatherly, L.G., Hodges, H., Eds.; CRC Press: Boca Raton, FL, USA, 1999; pp. 41-47.

15. Setiyono, T.D.; Weiss, A.; Specht, J.; Bastidas, A.M.; Cassman, K.G.; Dobermann, A. Understanding and modeling the effect of temperature and daylength on soybean phenology under high-yield conditions. Field Crops Res. 2007, 100, 257-271. [CrossRef]

16. George, T.; Bartholomew, D.P.; Singleton, P.W. Effect of temperature and maturity group on phenology of field grown nodulating and nonnodulating soybean isolines. Biotronics 1990, 19, 49-59.

17. Heatherly, L.G.; Elmore, R.W. Managing inputs for peak production. In Soybeans: Improvement, Production, and Uses, Agronomy Monograph; Boerma, H.R., Specht, J.E., Eds.; American Society of Agronomy, Crop Science Society of America and Soil Science Society of America: Madison, WI, USA, 2004; pp. 451-536.

18. Salmerón, M.; Gbur, E.E.; Bourland, F.M.; Earnest, L.; Golden, B.R.; Purcell, L.C. Soybean maturity group choices for maximizing radiation interception across planting dates in the Midsouth United States. Agron. J. 2015, 107, 2132-2142. [CrossRef]

19. Salmeron, M.; Gbur, E.E.; Bourland, F.M.; Buehring, N.W.; Earnest, L.; Fritschi, F.B.; Golden, B.R.; Hathcoat, D.; Lofton, J.; Miller, T.D. Soybean maturity group choices for early and late plantings in the Midsouth. Agron. J. 2014, 106, 1893-1901. [CrossRef]

20. Bruns, H.A. Planting date, rate, and twin-row vs. single-row soybean in the Mid-South. Agron. J. 2011, 103, 1308-1313. [CrossRef]

21. Grichar, W.J.; Biles, S.P. Response of soybean to early-season planting dates along the upper Texas Gulf Coast. Int. J. Agron. 2014, 4, 190-195. [CrossRef]

22. Singh, B.; Reddy, K.R.; Redoña, E.D.; Walker, T. Developing a screening tool for osmotic stress tolerance classification of rice cultivars based on in vitro seed germination. Crop Sci. 2017, 57, 387-394. [CrossRef]

23. Singh, B.; Reddy, K.R.; Redoña, E.D.; Walker, T. Screening of rice cultivars for morpho-physiological responses to early-season soil moisture stress. Rice Sci. 2017, 24, 322-335. [CrossRef]

24. Cober, E.R.; Curtis, D.F.; Stewart, D.W.; Morrison, M.J. Quantifying the effects of photoperiod, temperature and daily irradiance on flowering time of soybean isolines. Plants 2014, 3, 476-497. [CrossRef]

25. Kurosaki, H.; Yumoto, S. Effects of low temperature and shading during flowering on the yield components in soybeans. Plant Prod. Sci. 2003, 6, 17-23. [CrossRef]

26. EWuebker, F.; Mullen, R.E.; Koehler, K. Flooding and temperature effects on soybean germination. Crop Sci. 2001, 41, 1857-1861. [CrossRef]

27. Singh, B.; Norvell, E.; Wijewardana, C.; Wallace, T.; Chastain, D.; Reddy, K.R. Assessing morphological characteristics of elite cotton lines from different breeding programmes for low temperature and drought tolerance. J. Agron. Crop Sci. 2018, 204, 467-476. [CrossRef]

28. Wijewardana, C.; Hock, M.; Henry, B.; Reddy, K.R. Screening corn hybrids for cold tolerance using morphological traits for early-season seeding. Crop Sci. 2015, 55, 851-867. [CrossRef] 
29. Khan, M.A.; Gemenet, D.C.; Villordon, A. Root system architecture and abiotic stress tolerance: Current knowledge in root and tuber crops. Front. Plant Sci. 2016, 7, 1584. [CrossRef] [PubMed]

30. Reddy, K.R.; Brand, D.; Wijewardana, C.; Gao, W. Temperature effects on cotton seedling emergence, growth, and development. Agron. J. 2017, 109, 1287-1379. [CrossRef]

31. Brand, D.; Wijewardana, C.; Gao, W.; Reddy, K.R. Interactive effects of carbon dioxide, low temperature, and ultraviolet-B radiation on cotton seedling root and shoot morphology and growth. Front. Earth Sci. 2016, 10, 607-620. [CrossRef]

32. Reddy, K.R.; Read, J.J.; Baker, J.T.; McKinion, J.M.; Tarpley, L.; Hodges, H.F.; Reddy, V.R. Soil-Plant-Atmosphere-Research (SPAR) facility: A tool for plant research and modeling. Biotronics 2001, 30, 27-50.

33. Koti, S.; Reddy, K.R.; Reddy, V.R.; Kakani, V.G.; Zhao, D. Interactive effects of carbon dioxide, temperature, and ultraviolet-B radiation on soybean (Glycine max L.) flower and pollen morphology, pollen production, germination, and tube lengths. J. Exp. Bot. 2004, 56, 725-736. [CrossRef]

34. Edwards, J.T.; Purcell, L.C. Soybean yield and biomass responses to increasing plant population among diverse maturity groups. Crop Sci. 2005, 45, 1770-1777. [CrossRef]

35. Wegerer, R.; Popp, M.; Hu, X.; Purcell, L. Soybean maturity group selection: Irrigation and nitrogen fixation effects on returns. Field Crops Res. 2015, 180, 1-9. [CrossRef]

36. Salmerón, M.; Gbur, E.E.; Bourland, F.M.; Buehring, N.W.; Earnest, L.; Fritschi, F.B.; Golden, B.R.; Hathcoat, D.; Lofton, J.; McClure, A.T. Yield response to planting date among soybean maturity groups for irrigated production in the US Midsouth. Crop Sci. 2016, 56, 747-759. [CrossRef]

37. Anderson, J.M.; Chow, W.S.; Park, Y.-I. The grand design of photosynthesis: Acclimation of the photosynthetic apparatus to environmental cues. Photosynth. Res. 1995, 46, 129-139. [CrossRef] [PubMed]

38. Fuchs, M. Infrared measurement of canopy temperature and detection of plant water stress. Theor. Appl. Climatol. 1990, 42, 253-261. [CrossRef]

39. Bolger, T.P.; Upchurch, D.R.; McMichael, B.L. Temperature effects on cotton root hydraulic conductance. Environ. Exp. Bot. 1992, 32, 49-54. [CrossRef]

(C) 2019 by the authors. Licensee MDPI, Basel, Switzerland. This article is an open access article distributed under the terms and conditions of the Creative Commons Attribution (CC BY) license (http:/ / creativecommons.org/licenses/by/4.0/). 